AEI-1999-36

MIT-CTP-2927

hep-th/0002127

\title{
Duality and Weyl Symmetry of 7-brane Configurations
}

\author{
Tamás Hauer \\ Max-Planck-Institut für Gravitationsphysik, \\ Albert-Einstein-Institut, D-14476 Golm, Germany. \\ Amer Iqbal and Barton Zwiebach \\ Center for Theoretical Physics, \\ Massachusetts Institute of Technology, \\ Cambridge, Massachusetts 02139, U.S.A. \\ E-mail: hauer@aei-potsdam.mpg.de, amer@mit.edu, zwiebach@mitlns.mit.edu
}

February 2000

\begin{abstract}
Extending earlier results on the duality symmetries of three-brane probe theories we define the duality subgroup of $S L(2, \mathbb{Z})$ as the symmetry group of the background 7branes configurations. We establish that the action of Weyl reflections is implemented on junctions by brane transpositions that amount to exchanging branes that can be connected by open strings. This enables us to characterize duality groups of brane configurations by a map to the symmetry group of the Dynkin diagram. We compute the duality groups and their actions for all localizable 7-brane configurations. Surprisingly, for the case of affine configurations there are brane transpositions leaving them invariant but acting nontrivially on the charges of junctions.
\end{abstract}




\section{Introduction}

The four dimensional $\mathcal{N}=2$ supersymmetric $S U(2)$ Yang-Mills theory with $N_{f}=4$ is a theory with full $S L(2, \mathbf{Z})$ duality symmetry and global so(8) symmetry [1]. The duality symmetry has nontrivial implications for the dyonic spectrum of the theory. In fact the dyons fall into the $\mathbf{8}_{\mathbf{v}}, \mathbf{8}_{\mathbf{s}}$ or $\mathbf{8}_{\mathbf{c}}$ representations according to their dyonic charges $(p, q)$. In addition, duality transformations permute such representations. This action can be characterized nicely as a homomorphism from the duality group $S L(2, \mathbf{Z})$ to the permutation group on three objects, the group of graph automorphisms of the so(8) Dynkin diagram.

This quantum field theory can be viewed as living on a D3 brane probe of a 7-brane background [2, 3]. The 7-brane background includes the six 7-branes that compose the $D_{4}$ singularity of the Kodaira classification. Both the duality symmetry of the theory, and its interaction with the $D_{4}$ symmetry carried by the branes can be understood simply in terms

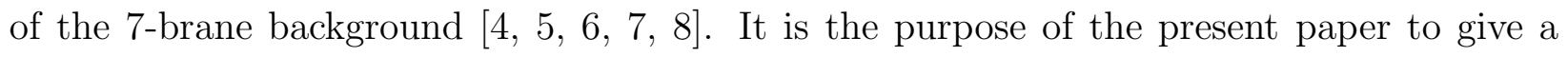
precise definition of the duality group associated to an arbitrary 7-brane configuration, and a description of its interaction with the Lie algebra carried by the configuration. Partial results were given in [6] where the duality group of a brane configuration with monodromy $K \in S L(2, \mathbf{Z})$ was thought to be simply the stabilizer subgroup of $K$ in $S L(2, \mathbf{Z})$. While correct for many cases, this is not always true and further conditions must be satisfied.

The duality symmetry of the four-dimensional effective theory is the remnant of the $S L(2, \mathbf{Z})$ symmetry of IIB string theory. Consider the simplest case of a D3 in the vicinity of D7branes. The three-brane is $S L(2, \mathbf{Z})$-symmetric while the D7-branes are left invariant by the subgroup generated by $T \in S L(2, \mathbf{Z})$; therefore the effective theory on the D3 still carries this subgroup as a duality symmetry (a trivial one because the theory does not have magnetic states). When 7 -branes of different charges are involved only $\mathbb{1}$ and $-\mathbb{1} \in S L(2, \mathbf{Z})$ leave each brane invariant. Those nontrivial transformations, however, which map the charges of the 7-branes to each other, do act as a duality symmetry of the effective theory because the transformed configuration is indistinguishable from the original one (although the position in the moduli space changes in general). The subtle task of identifying those $S L(2, \mathbf{Z})$ matrices which permute the charges of a given 7-brane configuration will be one subject of our present work. The difficulty arises because there is a large redundancy in characterizing a background by a list of 7-brane charges. To this end we will define the equivalence classes of configurations along the lines of [9, 10]: equivalent 7-brane setups are related to each other by the process of moving branch cuts of 7 -branes through each other. Then we look for $S L(2, \mathbf{Z})$ transformations which map a given 7-brane configuration to another one in its equivalence class.

Having found the "unbroken part" of $S L(2, \mathbf{Z})$ one should identify how this duality symmetry 
acts on the spectrum of the theory. As is well known from the Seiberg-Witten example, the duality group acts through the automorphism group of the root lattice of the algebra carried by the 7-branes. Such automorphisms are of two kinds: those related to the automorphisms of the Dynkin diagram, and those arising from Weyl reflections. In elucidating the general theory we have found it necessary to identify how Weyl symmetries are represented on the 7-brane configurations.

The states of D3 brane probe theories are strings or string junctions stretched between the D3 and (some of the) background 7-branes. When the 7-branes are on top of each other, the $7+1$ dimensional low energy model is a Yang-Mills theory with some gauge algebra $\mathcal{G}$ while the spectrum of the 4-dimensional D3-theory furnishes a representation of $\mathcal{G}$ which is a global symmetry. When the 7-branes do not coincide (and many times they cannot) the states are no longer degenerate but $\mathcal{G}$ is still a useful spectrum generating algebra. In particular states constituting the orbits of the Weyl group of $\mathcal{G}$ are of the same multiplicity. Indeed, Weyl reflections derive from an ambiguity in the choice of a base of simple roots, and states related by Weyl reflections are really physically equivalent.

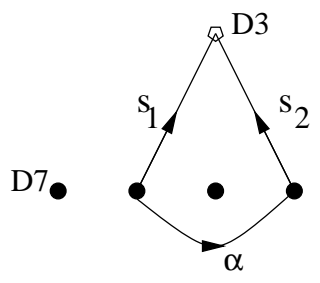

(a)

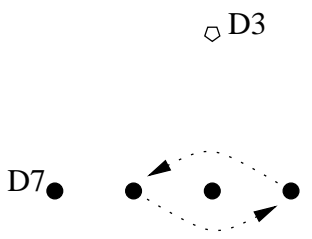

(b)

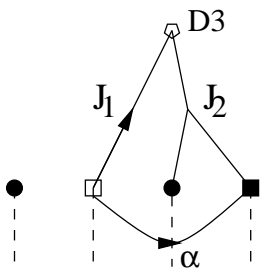

(c)

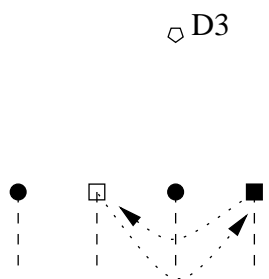

(d)

Figure 1: Weyl transformations correspond to branch cut moves of the 7-brane configuration.

How do Weyl transformations act on junctions? Consider a configuration of D7-branes. Fig. [(a) shows two strings, $\mathbf{s}_{1}$ and $\mathbf{s}_{2}$ which are in the fundamental representation of $s u(n)$ and are mapped to each other by the Weyl reflection with the root corresponding to $\alpha$. This is the case because using familiar intersection rules and composition of open strings we see that $\mathbf{s}_{1}+\left(\mathbf{s}_{1} \cdot \boldsymbol{\alpha}\right) \boldsymbol{\alpha}=\mathbf{s}_{1}-\boldsymbol{\alpha}=\mathbf{s}_{2}$. Physically, this Weyl reflection is implemented by the exchange process of the two D7-branes (see Fig. 1](b)), giving us an identical theory in which the two states $\mathbf{s}_{1}$ and $\mathbf{s}_{2}$ are replaced with each other. The cases involving mutually nonlocal 7-branes like the one in Fig. 1. (c) are treated similarly except that one should be careful when exchanging 7-branes because the path along which they are moved is relevant. Anticipating a more extended discussion later in the paper, we note that the rules for transpositions of 7-branes imply that the transformation shown in Fig. 目(d) leaves the brane configuration invariant. In the process of transposition the two junctions $\mathbf{J}_{1}$ and $\mathbf{J}_{2}=\mathbf{J}_{1}+\boldsymbol{\alpha}\left(\mathbf{J}_{1} \cdot \boldsymbol{\alpha}\right)$ can be shown to transform into each other as a consequence of prong creation taking place when 7-branes move through strings. Thus $\mathbf{J}_{1}$ and $\mathbf{J}_{2}$, while not equivalent junctions, represent 
states that are physically indistinguishable. We show generally that Weyl reflections act on junctions as automorphisms of the junction lattice generated by exchanging 7-branes through a path along which they are mutually local.

Weyl and duality transformations are different in nature: the former is an automorphism of the junction lattice mapping states into states with the same asymptotic charges, while the latter, acting through an element of $S L(2, \mathbf{Z})$ can change the asymptotic charges of junctions. In addition to this action, duality transformations are characterized by the kind of transformations they generate on the Lie-algebraic data characterizing the junctions. We show that these amount to automorphisms of the root lattice $Q$ of the Lie algebra carried by the 7-branes. Sometimes duality transformations give Weyl transformations of the root lattice, in which case they relate states with possibly different asymptotic charges, but having essentially equivalent Lie-algebraic data. On the other hand, some duality transformations map to automorphisms that correspond to symmetries of the Dynkin diagram. Such transformations are never Weyl reflections, and relate states in different Weyl orbits or different representations.

We define the duality group $\mathcal{D}$ of a brane configuration as the subgroup of $S L(2, \mathbf{Z})$ whose effect on the brane configuration can be undone by crossing transformations. A 7-brane background carrying a Lie algebra $\mathcal{G}$ has a duality group $\mathcal{D}$ that interacts nontrivially with $\mathcal{G}$ when the symmetry group $\Gamma$ of the Dynkin diagram of $\mathcal{G}$ is nontrivial. We will see that in general there is no canonical map $\mathcal{D} \mapsto \Gamma$ because of the surprising fact that invariant transpositions, transpositions that leave the brane configuration invariant, do not always act as Weyl transformations on the Lie algebraic data of the junction. Sometimes they may act as some outer automorphism of the root lattice. Since $\Gamma$ precisely represents outer automorphisms, the lack of a canonical map simply results because after an $S L(2, \mathbf{Z})$ duality, the restoring crossing transformation is ambiguous up to invariant transpositions.

If the action of the set invariant transpositions on the Lie algebraic data induces a Weyl transformation then the above map is well defined. This is the case for all the configurations realizing finite algebras. Particularly interesting are the cases of configurations carrying affine exceptional symmetries [11, 12, 13, 14, 15]. In these cases the Dynkin diagrams typically have nontrivial automorphism groups and therefore dualities interact rather nontrivially with the Lie algebra data. Also for these configurations not all invariant transpositions induce Weyl transformations on the Lie algebraic data of a junction. Therefore for these configurations the above map is only well defined with respect to a particular set of transpositions used to undo the $S L(2, \mathbf{Z})$ transformations. In this paper we compute the duality groups $\mathcal{D}$ and the maps to the symmetry groups $\Gamma$ of Dynkin diagrams for all brane configurations that can be localized in a compactification of type IIB string theory (see [14]).

Let us mention two questions that we have not discussed in this paper. While the action 
of dualities on root junctions determines fully the action of duality on weight junctions for finite algebras, in the case of affine Kac-Moody algebras more work is necessary to understand how dualities act on junctions that represent general weight vectors. Thus, for the affine exceptional configurations discussed here, our results are restricted to junctions in the root lattice. Second, we have not characterized the duality action for the configuration giving the Lie algebra $\widehat{E}_{9}$. Since this is not an affine Kac-Moody algebra the automorphism group of its root lattice appears to be unfamiliar.

In this paper, as in our previous ones [10, 12] we have focused on the symmetry aspects of 7-brane configurations. Another line of works dealing mostly with tests of F-theory/heterotic duality has been reviewed in [16] and some recent works of interest are [17, 18].

The paper is arranged as follows. In section 2 we define equivalent 7-brane backgrounds and the duality group of a 7-brane configuration. Section 3 introduces the Weyl transformations in 7-brane language. In section 4 we discuss the homomorphism between the duality (effective) group and the automorphism group of the Dynkin diagram of the underlying algebra. In sections 5 and 6 we systematically compute duality groups and homomorphisms for 7-brane configurations corresponding to finite and affine algebras, respectively.

\section{Brane configurations, duality and crossing transfor- mations}

The purpose of this section is to give a precise definition and begin the characterization of the duality group $\mathcal{D}(w)$ of a configuration $w$ of 7-branes. The duality group is a subgroup of $S L(2, \mathbf{Z})$ leaving the brane configuration invariant in a sense we describe in detail. Roughly,

an $S L(2, \mathbf{Z})$ element belongs to the duality group when its action on the branes can be undone by a crossing transformation, that is successive operations of brane transpositions. We show that both the monodromy $K(w)$ of a configuration and the $(-\mathbb{1})$ matrix belong to the duality group $\mathcal{D}(w)$. We also examine how crossing transformations act on junctions, and that leads to the understanding of how dualities act on junctions. In particular we show that the duality transformation $K(w)$ can be defined to leave invariant junctions having zero asymptotic charge. The general characterization of duality groups by their action on junctions is left for the next section.

Our presentation here will be formal for the sake of precision and brevity. Certainly the idea of the duality group of a brane configuration is not new. The duality groups of SeibergWitten $N=2 \mathrm{SYM}$ theories, in particular those of the $N_{f} \leq 4 S U(2)$ theories have been studied in detail and correspond to the duality groups of the 7-brane configurations where 
these four-dimensional theories arise on a D3 brane probe. A definition of the duality group for $D_{N>4}$ and $E_{6}, E_{7}, E_{8}$ was given in [6]. The present one is a refined version of that definition, applicable to other brane configurations as well.

We define what we mean by a 7-brane configuration keeping the canonical picture in mind: the branes are arranged along the real axis with their branch cuts going downwards and the monodromies are listed in the order the branes appear from left to right [9, 5.

\subsection{Definitions and properties}

- Definition: 7-brane configuration or simply a configuration is defined to be a word of $S L(2, \mathbf{Z})$ matrices conjugate to $T^{-1}$. That is, a 7 -brane is characterized by the monodromy of the axion-dilaton field $\tau$ around it. Via introduction of branch cuts $\tau$ is made single valued and $[A]$ stands for a 7 -brane with a cut where $\tau$ jumps by $A \in S L(2, \mathbf{Z})$. To define a 7-brane background we list the branes with cuts going downwards from left to right. The set of all configurations consisting of $n 7$-branes we denote as $\mathcal{C}_{n}$ :

$$
\begin{aligned}
\mathcal{C}_{n} \ni w & =\left[A_{1}\right]\left[A_{2}\right] \ldots\left[A_{n}\right] \quad A_{i}=g_{i} T^{-1} g_{i}^{-1}, \quad g_{i} \in S L(2, \mathbf{Z}), \\
K(w) & \equiv A_{n} \ldots A_{2} A_{1} \in S L(2, \mathbf{Z}) .
\end{aligned}
$$

$K(w)$ is the overall monodromy associated with the configuration $w$. The purpose of the square bracket is to distinguish words of matrices from products of them, i.e. $\left[A_{1} A_{2}\right] \in \mathcal{C}_{1}$ denotes a one-letter word of the matrix $A=A_{1} A_{2}$ while $\left[A_{1}\right]\left[A_{2}\right] \in \mathcal{C}_{2}$ is a two letter word made of these two.

- Definition: $S L(2, \mathbf{Z})$ action on $\mathcal{C}_{n}$. $S L(2, \mathbf{Z})$ symmetry of IIB transforms $\tau$ and as a consequence the monodromy of a 7 -brane is conjugated by this transformation $g \in S L(2, \mathbf{Z})$. The image of a configuration is simply the word of the transformed matrices:

$$
S L(2, \mathbf{Z}) \ni g: \mathcal{C}_{n} \rightarrow \mathcal{C}_{n} \quad\left[A_{1}\right]\left[A_{2}\right] \ldots\left[A_{n}\right] \mapsto\left[g A_{1} g^{-1}\right]\left[g A_{2} g^{-1}\right] \ldots\left[g A_{n} g^{-1}\right] .
$$

- Definition: Transposition of 7-branes. The position of the branch cuts of the 7branes are unphysical and they can be relocated by performing an immaterial $S L(2, \mathbf{Z})$ transformation on all physical parameters in a selected region. In particular the relative order of the cuts can be changed but the 7-branes through which a branch cut is moved are subject to the same $S L(2, \mathbf{Z})$ transformation. The elementary change of a configuration is when a cut of a brane is moved through its left hand $(P)$ or right hand 
$\left(P^{-1}\right)$ neighbor. The transposition of the $m$-th and $m+1$-th letter of a word is thus performed by the following rule [9]:

$$
\begin{aligned}
& P_{m}: \quad \ldots\left[A_{m}\right]\left[A_{m+1}\right] \ldots \longmapsto \ldots\left[A_{m+1}\right]\left[A_{m+1} A_{m} A_{m+1}^{-1}\right] \\
& P_{m}^{-1}: \ldots\left[A_{m}\right]\left[A_{m+1}\right] \ldots \longmapsto \ldots\left[A_{m}^{-1} A_{m+1} A_{m}\right]\left[A_{m}\right] \ldots
\end{aligned}
$$

Notice that $A_{m}^{-1} A_{m+1} A_{m}$ is conjugate to $T^{-1}$ if $A_{m+1}$ is, therefore the result is indeed in $\mathcal{C}_{n}$. Also note that in general $P_{m}^{2}$ is non trivial.

- Property 1: The transpositions satisfy the following Braid group relations:

$$
P_{m} P_{m^{\prime}}=P_{m^{\prime}} P_{m}, \text { if }\left|m-m^{\prime}\right|>1, \quad P_{m} P_{m+1} P_{m}=P_{m+1} P_{m} P_{m+1}
$$

- Definition: The Group of crossing transformations $\mathcal{B}_{N}$ (corresponding to branch cut moves) is defined by its action on $\mathcal{C}_{n}$. This group is generated by all transpositions $\left\{P_{m}\right\}_{m=1}^{n-1}$ subject to the constraint (2.4).

- Property 2: The $S L(2, \mathbb{Z})$ transformations as defined in (2.2) commute with the transpositions. If $g$ denotes an $S L(2, \mathbb{Z})$ transformation and $b$ a series of transpositions, we have $g(b(w))=b(g(w))$. To prove this it suffices to examine the case when $b$ is a single transposition, say $P_{1}$ :

$$
\begin{array}{ccc}
{\left[A_{1}\right]\left[A_{2}\right]} & \stackrel{P_{1}}{\longrightarrow} & {\left[A_{2}\right]\left[A_{2} A_{1} A_{2}^{-1}\right]} \\
\downarrow & & \downarrow g \\
{\left[g A_{1} g^{-1}\right]\left[g A_{2} g^{-1}\right]} & \stackrel{P_{1}}{\longrightarrow} & {\left[g A_{2} g^{-1}\right]\left[g A_{2} A_{1} A_{2}^{-1} g^{-1}\right]}
\end{array}
$$

- Definition: Equivalence group. As explained before, if two 7-brane configurations differ by either an overall $S L(2, \mathbf{Z})$ or by crossing transformations, they are physically identical. The need for distinguishing between configurations up to this equivalence motivates the following definition. The equivalence group of $\mathcal{C}_{n}$ is the direct product of the two groups: $\mathcal{E}_{n} \equiv S L(2, \mathbf{Z}) \times \mathcal{B}_{n}$. The action of elements of $\mathcal{E}_{n}$ on $\mathcal{C}_{n}$ is welldefined due to the commutativity of the actions of the two factors, $(g, b) w=g(b(w))=$ $b(g(w))$. The product on this set is defined by $\left(g_{1}, b_{1}\right)\left(g_{2}, b_{2}\right)=\left(g_{1} g_{2}, b_{1} b_{2}\right)$ where $g_{i} \in S L(2, \mathbf{Z}), b_{i} \in \mathcal{B}_{n}$.

Acting with an element of $\mathcal{C}_{n}$ changes the 7-branes in general. There is however a subgroup of $\mathcal{C}_{n}$ which leaves the configuration invariant and thus acts as a symmetry of the D3 probe theory. Let us therefore define 
- Definition: The symmetry group $\mathcal{S}(w) \subset \mathcal{E}_{n}$ of the configuration $w$ is given by

$$
\mathcal{S}(w) \equiv\left\{(g, b) \in \mathcal{E}_{n} \mid(g, b) w=w\right\} .
$$

$\mathcal{S}$ is manifestly a group, indeed a very large one because for a given $g$ there typically are many choices of $b$ satisfying the condition $(g, b) w=w$. For a given $g$ the transformation $b$ is not unique because there are crossing transformations $b$ that leave $w$ invariant. These form a normal subgroup $\mathcal{H}(w)$ of $\mathcal{S}(w)$ :

$$
\mathcal{H}(w) \equiv\{(\mathbb{1}, b) \in \mathcal{S}(w)\} .
$$

The subgroup of the IIB duality group $S L(2, \mathbf{Z})$ which preserves $w$ consists of all those elements $g \in S L(2, \mathbf{Z})$ appearing in $\mathcal{S}(w)$. This means forgetting about the compensating transformation $b$ and thus leads one to define:

- Definition: The duality group $\mathcal{D}(w)$ of a configuration $w$ is defined as

$$
\mathcal{D}(w) \equiv \mathcal{S}(w) / \mathcal{H}(w) \cong\left\{g \in S L(2, \mathbb{Z}) \mid \exists b \in \mathcal{B}_{n} \text { such that }(g, b) w=w\right\} .
$$

This is clearly the subgroup of $S L(2, \mathbb{Z})$ whose elements leave $w$ invariant after the action of a suitable crossing transformation.

- Proposition: $K(w) \in \mathcal{D}(w)$ : the duality group necessarily contains the overall monodromy. In addition, $-\mathbb{1} \in \mathcal{D}(w)$.

Proof: Consider the configuration $w=\left[A_{1}\right]\left[A_{2}\right] \ldots\left[A_{n}\right]$, with $K_{n}=K(w)=A_{n} \ldots A_{1}$. Also define $K_{i}=A_{i} \ldots A_{1}$, for $1 \leq i \leq n$. We will explicitly construct an element $b$ of $\mathcal{B}_{n}$ in terms of transpositions which satisfies $\left(K_{n}, b\right) w=w$. Let us perform the $S L(2, \mathbb{Z})$ transformation with $K_{n}^{-1}$ :

$$
\left[A_{1}\right]\left[A_{2}\right] \ldots\left[A_{n}\right] \stackrel{K_{n}^{-1}}{\longrightarrow}\left[K_{n}^{-1} A_{1} K_{n}\right]\left[K_{n}^{-1} A_{2} K_{n}\right] \ldots\left[K_{n}^{-1} A_{n} K_{n}\right] .
$$

As the first step, apply the product $\left(P_{1} \ldots P_{n-1}\right) \in \mathcal{B}_{N}$ to the rhs of $(2.9)$ : this corresponds to moving the cut of the rightmost brane through the rest of them; then repeat this process $n-1$ times. We claim that not only the order of the branes is restored but their charges are transformed back to the original ones:

$$
\begin{aligned}
\stackrel{P_{1} \ldots P_{n-1}}{\longrightarrow} & {\left[K_{n}^{-1} A_{n} K_{n}\right]\left[K_{n}^{-1} A_{n} A_{1} A_{n}^{-1} K_{n}\right] \ldots\left[K_{n}^{-1} A_{n} A_{n-1} A_{n}^{-1} K_{n}\right] } \\
& =\left[K_{n-1}^{-1} A_{n} K_{n-1}\right]\left[K_{n-1}^{-1} A_{1} K_{n-1}\right] \ldots\left[K_{n-1}^{-1} A_{n-1} K_{n-1}\right] \\
\stackrel{P_{1} \ldots P_{n-1}}{\longrightarrow} & {\left[K_{n-1}^{-1} A_{n-1} K_{n-1}\right]\left[K_{n-1}^{-1} A_{n-1} A_{n} A_{n-1}^{-1} K_{n-1}\right] \ldots\left[K_{n-1}^{-1} A_{n-1} A_{n-2} A_{n-1}^{-1} K_{n-1}\right] } \\
& =\left[K_{n-2}^{-1} A_{n-1} K_{n-2}\right]\left[K_{n-2}^{-1} A_{n} K_{n-2}\right] \ldots\left[K_{n-2}^{-1} A_{n-2} K_{n-2}\right] \\
& \vdots \\
\stackrel{P_{1} \ldots P_{n-1}}{\longrightarrow} & {\left[A_{1}\right]\left[A_{2}\right] \ldots\left[A_{n}\right] . }
\end{aligned}
$$


Thus we proved that the overall monodromy is indeed in the duality group, that is

$$
\left(K(w),\left(P_{1} P_{2} \ldots P_{n-1}\right)^{-n}\right) w=\left(K(w)^{-1},\left(P_{1} P_{2} \ldots P_{n-1}\right)^{n}\right) w=w .
$$

For the transformation $g=-\mathbb{1}$ we simply note that this transformation does not change the word describing the configuration since for each brane $\left[A_{i}\right] \rightarrow\left[g A_{i} g^{-1}\right]=\left[A_{i}\right]$. This transformation is clearly in the duality group and since it plainly leaves the 7 -brane configuration invariant it does not need to be accompanied by brane transpositions ( $b$ can be taken to be the identity in (2.8)).

\subsection{The action on invariant charges}

Having seen how the $S L(2, \mathbf{Z})$ equivalence actions transform the 7 -brane configurations we would like to know the fate of junctions ending on these 7-branes. A junction is characterized by its invariant charges; the effective number of prongs on each 7-brane of the configuration [5]. The action of an overall $S L(2, \mathbf{Z})$ transformation is trivial: the charges of each string composing the junction transform as a doublet but the invariant charges do not change.

In general when one performs a crossing transformation, not only the 7-brane labels change but the invariant charges on those branes change as well. This is most easily seen from the active viewpoint: instead of relocating the cuts moving them through the 7-branes, we can move the 7-branes. Whenever a 7-brane in motion crosses a string segment, additional prongs on that brane might be created.

It suffices to determine how the charges change in the transposition of two consecutive 7branes, more complicated cases are considered by successive transpositions. Consider therefore a junction on a pair of 7 -branes $\left[K_{\mathbf{z}_{1}}\right]\left[K_{\mathbf{z}_{2}}\right] \equiv\left[\mathbf{z}_{1}\right]\left[\mathbf{z}_{2}\right]$ with invariant charges $\left[Q_{1}\right]\left[Q_{2}\right]$. Here we label the branes, as in [10] with their charge vector $\mathbf{z}=(p, q)$, in terms of which the corresponding $S L(2, \mathbf{Z})$ monodromy matrix is written as $K_{\mathbf{z}} \equiv \mathbb{1}+\mathbf{z z}^{T} S$. According to (2.3) the charges of the branes transform as $\left(s_{12} \equiv \mathbf{z}_{1} \times \mathbf{z}_{2}=p_{1} q_{2}-q_{1} p_{2}\right)$ :

$$
\begin{array}{lll}
P & :\left[\mathbf{z}_{1}\right]\left[\mathbf{z}_{2}\right] & \mapsto\left[\mathbf{z}_{2}\right]\left[K_{\mathbf{z}_{2}} \mathbf{z}_{1}\right]=\left[\mathbf{z}_{2}\right]\left[\mathbf{z}_{1}+s_{12} \mathbf{z}_{2}\right] \\
P^{-1}: & \left.\left[\mathbf{z}_{1}\right]\left[\mathbf{z}_{2}\right] \mapsto K_{\mathbf{z}_{1}}^{-1} \mathbf{z}_{2}\right]\left[\mathbf{z}_{1}\right]=\left[\mathbf{z}_{2}+s_{12} \mathbf{z}_{1}\right]\left[\mathbf{z}_{1}\right] .
\end{array}
$$

The action on the invariant charges can be determined by looking at how many prongs are created/annihilated on each 7-brane in the canonical presentation [5], but charge conservation alone gives the answer too:

$$
\begin{array}{lllll}
P & : & {\left[Q_{1}\right]\left[Q_{2}\right]} & \mapsto & {\left[Q_{2}-s_{12} Q_{1}\right]\left[Q_{1}\right]} \\
P^{-1} & : & {\left[Q_{1}\right]\left[Q_{2}\right]} & \mapsto & {\left[Q_{2}\right]\left[Q_{1}-s_{12} Q_{2}\right]}
\end{array}
$$


Appending the invariant charges as superscripts to the branes, the complete action for both transpositions is:

$$
\begin{aligned}
& {\left[\mathbf{z}_{1}\right]^{Q_{1}}\left[\mathbf{z}_{2}\right]^{Q_{2}} \stackrel{P}{\longmapsto}\left[\mathbf{z}_{2}\right]^{Q_{2}-s_{12} Q_{1}}\left[\mathbf{z}_{1}+s_{12} \mathbf{z}_{2}\right]^{Q_{1}}} \\
& {\left[\mathbf{z}_{1}\right]^{Q_{1}}\left[\mathbf{z}_{2}\right]^{Q_{2}} \stackrel{P^{-1}}{\longmapsto}\left[\mathbf{z}_{2}+s_{12} \mathbf{z}_{1}\right]^{Q_{2}}\left[\mathbf{z}_{1}\right]^{Q_{1}-s_{12} Q_{2}} .}
\end{aligned}
$$

The crossing transformation that restores the original brane configuration after application of the monodromy $K$ (see (2.11)) has an important property: it does not change the invariant charges of junctions with zero asymptotic charge. This is best seen by visualizing the motion of the 7-branes. In Fig. 2 we show the effect of the cyclic transformation $P_{1} \ldots P_{n-1}$ : it corresponds to moving the rightmost 7-brane above the rest to the left. If a junction has no asymptotic charge, this 7-brane does not cross any string segment along the transformation and therefore the invariant charge of any of 7-branes does not change. Performing this transformation $n$ times rearranges the 7-branes in the original order and although their monodromies change, the invariant charges of any given junction without asymptotic charge remain the same.
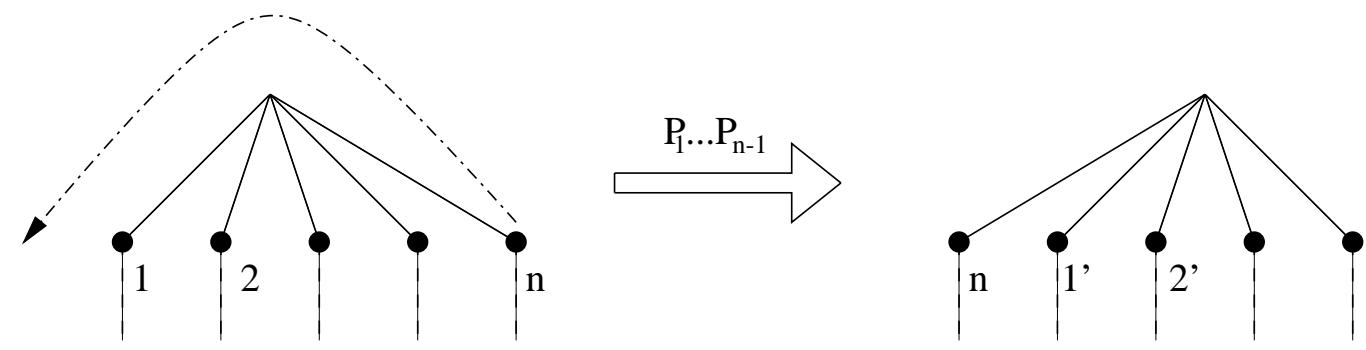

Figure 2: Action of $P_{1} \ldots P_{n-1}$ on a 7-brane configuration with a string junction of no asymptotic charge. While moving the rightmost brane, the $[p, q]$-charges of the branes change but the invariant charges of the junction do not.

We note in passing that while the $g=-\mathbb{1} \in \mathcal{D}(w)$ transformation does not change the list of branes, the invariant charges of any junction will change sign.

\section{Implementing Lie algebra Weyl transformations}

In section 2 we introduced the group $\mathcal{H}(w)$ of invariant crossing transformations. The elements of this group are crossing transformations $b$ that leave the brane configuration $w$ invariant, namely, $b w=w$. It is the purpose of this section to understand some of the structure of $\mathcal{H}(w)$. 
We know that invariant crossing transformations act on junctions by shuffling their invariant charges, and therefore act on weight vectors of the Lie algebra carried by the branes. We will show that Weyl transformations of the Lie algebra can always be implemented at the level of junctions by invariant crossing transformations and this will take most of the work in the present section. Nevertheless, there are sometimes invariant transformations that do not correspond to Weyl transformations, but rather correspond to outer automorphisms of the root lattice of the algebra. This we found to be a surprise. We will give here a nontrivial example of this phenomenon.

It is useful to define the subgroup $\mathcal{H}_{W}(w)$ called the group of invariant crossing transformations of Weyl type. A transformation is said to belong to $\mathcal{H}_{W}(w)$ if its action on weights corresponding to junctions is a Weyl transformation. There are some special invariant transformations that do not change junctions at all. Such transpositions belong to $\mathcal{H}_{W}(w)$ since they imply a trivial identity Weyl transformation. We give an example of such transposition.

As we will see later in this paper, whenever $\mathcal{H}_{W}(w)$ coincides with $\mathcal{H}(w)$, namely, if all invariant transpositions are of Weyl type, the characterization of duality groups is very much simplified. This will be the case for finite algebras, but not the case for affine ones.

\subsection{Weyl transformations as invariant crossing transformations}

In this section our main objective is to prove that for any Weyl transformation of the Lie algebra $\mathcal{G}(w)$ carried by a 7-brane configuration $w$ there is a crossing transformation which implements this Weyl transformation at the level of junctions. This crossing transformation leaves the brane configuration invariant and simply acts on the invariant charges of junctions supported on the configuration. This action is such that the associated weight vectors undergo the desired Weyl transformation. We restrict our attention to the finite and affine Kac-Moody algebras that can be obtained on localizable brane configurations.

We begin by noting that Weyl transformations are generated by elementary reflections using the simple roots of the algebras in question. We also recall [19, 5, 11] that each simple root junction of the $\mathbf{A}^{N}, \mathbf{H}_{0 \leq N \leq 3}, \mathbf{D}_{N \geq 0}, \mathbf{E}_{6 \leq N \leq 8}$ and $\widehat{\mathbf{E}}_{0 \leq N \leq 8}$ configurations can be represented by an open string $\boldsymbol{\alpha}\left(\boldsymbol{\alpha}^{2}=-2\right)$ stretched between two possibly mutually non-local 7-branes.

We now claim that there is a rearrangement of the configuration, equivalent to a crossing transformation $b$, such that the root $\boldsymbol{\alpha}$ in question becomes an open string $b(\boldsymbol{\alpha})$ stretched between two mutually local 7-branes. This rearrangement corresponds to moving one of the two 7-branes on which $\boldsymbol{\alpha}$ ends along the path of $\boldsymbol{\alpha}$ itself until the brane is just to the side of other brane. This motion, for a particular example, is shown in Fig. 3. One can imagine the branch cut of the moving brane staying vertical, and one can see that indeed 
this motion simply corresponds to a sequence of transpositions of branes. After this motion, however, many of the branes of the configuration may have changed identity, and therefore the configuration has not been left invariant.
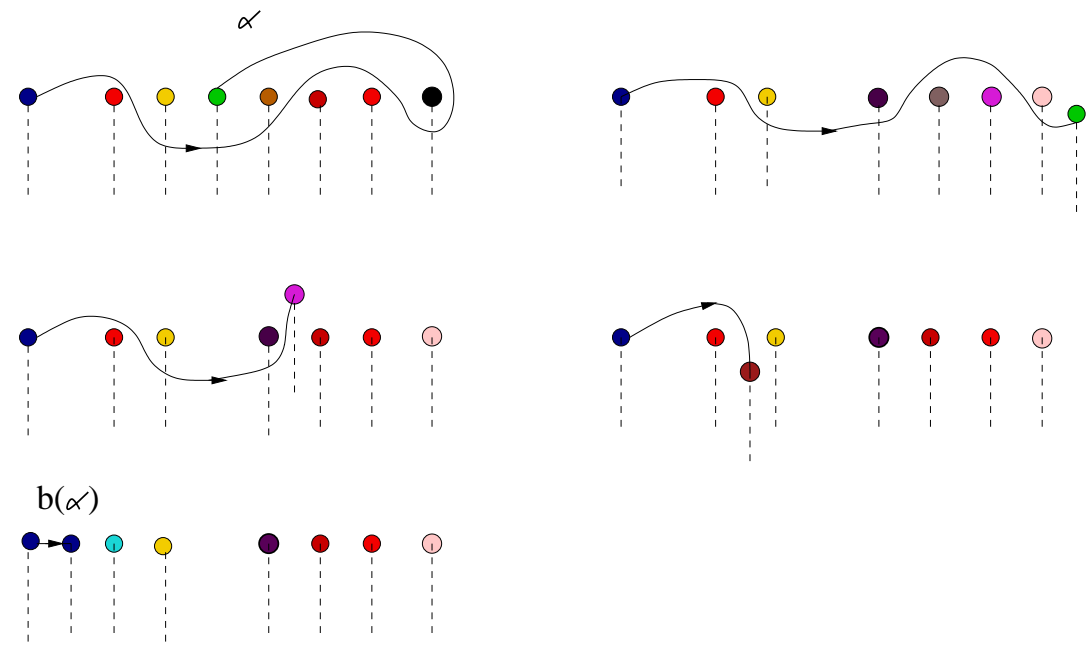

Figure 3: An open string between two 7-branes can be transformed into an open string between two mutually local 7-branes by rearranging the branes.

We now claim that the elementary Weyl reflection using the root $\boldsymbol{\alpha}$ is obtained by first doing the transpositions in $b$, then doing the transposition $P_{i}$ that interchanges the two mutually local 7-branes supporting the now short open string $b(\boldsymbol{\alpha})$, and then using the brane that sits where the first one ended to retrace backwards the path, this is simply done by applying $b^{-1}$. Two facts should be noted. First, the original open string $\boldsymbol{\alpha}$ changes direction, thus becoming $-\boldsymbol{\alpha}$ as one would expect for a Weyl transformation generated by $\boldsymbol{\alpha}$. Second, by retracing the path, all changes of brane labels that the first tracing of the path caused are compensated and the brane configuration is now left invariant. It is now left to show that this sequence of operations $\left(b^{-1} P_{i} b\right)$ performs the expected Weyl reflection on arbitrary junctions supported on the configuration. That is,

$$
\mathrm{W}_{\alpha}=b^{-1} P_{i} b: \mathbf{J} \rightarrow \mathbf{J}+(\mathbf{J} \cdot \boldsymbol{\alpha}) \boldsymbol{\alpha} .
$$

We first show explicitly that this formula holds in the special case when $\boldsymbol{\alpha}$ is an open string stretching between two adjacent mutually local branes $\left[\mathbf{z}_{\mathbf{i}}\right]$ and $\left[\mathbf{z}_{i+1}\right]$. In this case $\boldsymbol{\alpha}=\mathbf{z}_{\mathbf{i}}-\mathbf{z}_{\mathbf{i}+\mathbf{1}}$, and $W_{\alpha}=P_{i}$, just the exchange of the two branes $\left[\mathbf{z}_{\mathbf{i}}\right]$ and $\left[\mathbf{z}_{i+1}\right]$. Consider now a general junction supported on the configuration

$$
\mathbf{J}=\sum Q_{k} \mathbf{z}_{k}=\mathbf{z}_{\mathbf{i}} Q_{i}+\mathbf{z}_{\mathbf{i}+\mathbf{1}} Q_{i+1}+\cdots \quad \rightarrow \quad(\mathbf{J} \cdot \alpha)=Q_{i+1}-Q_{i}
$$


The exchange $P_{i}$ of the mutually local branes $\left[\mathbf{z}_{\mathbf{i}}\right]$ and $\left[\mathbf{z}_{\mathbf{i}+\mathbf{1}}\right]$ maps $\mathbf{J}$ to the new junction $\mathbf{J}^{\prime}$ defined as

$$
P_{i}: \mathbf{J} \rightarrow \mathbf{J}^{\prime}=\mathbf{z}_{\mathbf{i}} Q_{i+1}+\mathbf{z}_{\mathbf{i}+\mathbf{1}} Q_{i}+\cdots=\mathbf{J}+(\mathbf{J} \cdot \boldsymbol{\alpha}) \boldsymbol{\alpha},
$$

where use was made of the explicit expression for $\boldsymbol{\alpha}$ and of equation (3.2). This confirms our claim for this special case.

Let us now return to the general problem and compute the action of $b^{-1} P_{i} b$ on a general junction as follows

$$
\left(b^{-1} P_{i} b\right)(\mathbf{J})=b^{-1}\left[P_{i}(b(\mathbf{J}))\right]=b^{-1}[b(\mathbf{J})+(b(\mathbf{J}) \cdot b(\boldsymbol{\alpha})) b(\boldsymbol{\alpha})],
$$

where in the last step we used (3.3) where the role of $\boldsymbol{\alpha}$ is played here by the junction $b(\boldsymbol{\alpha})$ extending between the two mutually local branes that are transposed. Since $b^{-1} b$ equals the identity on any junction, and the intersection of two junctions is invariant under crossing transformations we find

$$
\left(b^{-1} P_{i} b\right)(\mathbf{J})=\mathbf{J}+(\mathbf{J} \cdot \boldsymbol{\alpha}) \boldsymbol{\alpha} .
$$

This completes our proof that Weyl transformations can be realized as crossing transformations that leave invariant the brane configuration. Our realization has been very specific, and while all such crossing transformations belong to $\mathcal{H}_{W}(w)$, they do not necessarily exhaust it, as we illustrate in section 3.2.

One can wonder if there is a useful notion of Weyl reflections of the junction lattice of a configuration that makes no reference to the Lie algebra carried by the configuration. For any junction $\boldsymbol{\alpha}$ such that $(\boldsymbol{\alpha} \cdot \boldsymbol{\alpha})=-2$ we could define

$$
\mathcal{W}_{\boldsymbol{\alpha}}(\mathbf{J})=\mathbf{J}+(\mathbf{J} \cdot \boldsymbol{\alpha}) \boldsymbol{\alpha} .
$$

This transformation preserves intersection numbers and it is therefore an automorphism of the junction lattice. The transformations generated by junctions $\boldsymbol{\alpha}$ of zero asymptotic charge correspond to the Lie algebraic Weyl transformations since such junctions are roots. On the other hand a transformation $\mathcal{W}_{\boldsymbol{\alpha}}$ generated by a junction $\boldsymbol{\alpha}$ with asymptotic charge will change the asymptotic charge of the junction on which it acts. The significance of such transformations is unclear since they generically map BPS junctions to non-BPS junctions.] Thus it may be that the only useful reflections of the junction lattice are those generated by roots of the Lie algebra carried by the configuration.

\footnotetext{
${ }^{1}$ Consider the $\widehat{\mathbf{E}}_{9}$ junction $\boldsymbol{\alpha}=\sum_{i=1}^{8} \mu_{i} \omega^{i}+2 \omega^{p}-\delta^{(0,1)}$, with the $E_{8}$ weight satisfying $\mu^{2}=2$. This junction has self-intersection minus two and non-zero asymptotic charge. Consider now $\mathcal{W}_{\boldsymbol{\alpha}}$ acting on the BPS junction $\delta^{(0,1)}$. One readily finds $\mathcal{W}_{\boldsymbol{\alpha}}\left(\delta^{(0,1)}\right)=2 \sum_{i=1}^{8} \mu_{i} \omega^{i}+4 \omega^{p}-\delta^{(0,1)}$. We know that a necessary condition for a junction $\mathbf{J}$ of asymptotic charge $(p, q)$ to be BPS is that $\mathbf{J} \cdot \mathbf{J} \geq-2+\operatorname{gcd}(p, q)$ [6]. Since $\left(\mathcal{W}_{\boldsymbol{\alpha}}\left(\delta^{(0,1)}\right)\right) \cdot\left(\mathcal{W}_{\boldsymbol{\alpha}}\left(\delta^{(0,1)}\right)\right)=0<-2+\operatorname{gcd}(4,0)$, it follows that $\mathcal{W}_{\boldsymbol{\alpha}}\left(\delta^{(0,1)}\right)$ cannot be BPS.
} 


\subsection{Further examples of invariant crossing transformations}

Above we presented a group of invariant crossing transformations that act on junctions via Weyl transformations of their Lie-algebraic data. This group, however, does not contain all invariant transpositions $\mathcal{H}(w)$ (see (2.7)) of the generic 7-brane configuration. Among the additional invariant crossing transformations there are ones that are of Weyl type as well as others which are not. In the following we give an example for each.

$\mathbf{D}_{\mathbf{N}}$ : For simplicity, consider first the case of $\mathbf{D}_{\mathbf{1}}$. This configuration has no root junction (a junction of self-intersection minus two and zero asymptotic charge) therefore there is no Weyl group of the usual kind, and there are no transpositions of the type discussed in the previous subsection. Nevertheless there is a nontrivial crossing transformation leaving the configuration invariant and thus belonging to $\mathcal{H}(w)$. This transformation leaves all charges unchanged, as will be shown now. Indeed,

$$
\begin{aligned}
& \mathbf{A}^{Q_{A}} \mathbf{B}^{Q_{B}} \mathbf{C}^{Q_{C}} \stackrel{P_{1}}{\longrightarrow} \mathbf{B}^{Q_{B}+Q_{A}} \mathbf{X}_{[\mathbf{0}, \mathbf{1}]}^{Q_{A}} \mathbf{C}^{Q_{C}} \stackrel{P_{2}}{\longrightarrow} \mathbf{B}^{Q_{B}+Q_{A}} \mathbf{C}^{Q_{C}+Q_{A}} \mathbf{A}^{-Q_{A}} \\
& \stackrel{P_{2}}{\longrightarrow} \mathbf{B}^{Q_{B}+Q_{A}} \mathbf{A}^{Q_{C}} \mathbf{X}_{[\mathbf{0}, \mathbf{1}]}^{Q_{C}+Q_{A}} \stackrel{P_{1}}{\longrightarrow} \mathbf{A}^{-Q_{A}+Q_{C}-Q_{B}} \mathbf{X}_{[\mathbf{2},-\mathbf{1}]}^{Q_{B}+Q_{A}} \mathbf{X}_{[\mathbf{0}, \mathbf{1}]}^{Q_{C}+Q_{A}} \\
& \stackrel{P_{1} P_{2} P_{2} P_{1}}{\longrightarrow} \mathbf{A}^{Q_{A}} \mathbf{X}_{[\mathbf{3},-\mathbf{1}]}^{Q_{C}} \mathbf{X}_{[-\mathbf{1}, \mathbf{1}]}^{2 Q_{C}-Q_{B}} \stackrel{P_{2}}{\longrightarrow} \mathbf{A}^{Q_{A}} \mathbf{B}^{Q_{B}} \mathbf{C}^{Q_{C}} .
\end{aligned}
$$

This transposition is obtained by first moving the A-brane around the $\mathbf{B C}$ branes twice and then moving the transformed $\mathbf{B}$-brane through the cut of the $\mathbf{C}$-brane. This brane configuration has this particular invariance because $T^{2} \in \mathcal{D}(w)$ (section 5.3), and therefore the $S L(2, \mathbb{Z})$ transformation $T^{2 k}$ induced on the $\mathbf{B C}$ system by the $\mathbf{A}$-brane can be undone by a transposition.

Now, we can turn to the case of $\mathbf{D}_{\mathbf{N}}$, where clearly the same transformation exists leaving the configuration and all invariant charges unchanged. This transformation is trivially of Weyl type in that it acts on the junctions's Lie algebraic data as the identity. On the other hand, it is also clear that this transformation cannot be obtained by composition of transformations that interchange branes connected by an open string. This is the case because all such open strings in $\mathbf{D}_{\mathbf{N}}$ join $\mathbf{A}$ branes and thus the $\mathbf{B}$ and $\mathbf{C}$ branes are never interchanged.

$\widehat{\tilde{\mathbf{E}}}_{1}$ : This 7 -brane configuration also does not admit any ordinary root junction. The only BPS junctions are multiples of the delta junction $\delta$ that encircles the configuration. Having no real roots we have no transformations generated by open strings. Surprisingly, there is an invariant transformation that actually changes the Lie algebraic data of junctions. In doing so it shows that elements of $\mathcal{H}(w)$ may generate in general nontrivial automorphisms that are not of Weyl type. It would be of interest to exhibit those explicitly for affine configurations

having a nontrivial Weyl group, but we focus here our attention to the case of $\widehat{\tilde{\mathbf{E}}}_{\mathbf{1}}$. The transformation in question is actually analogous to the one just considered above. We first 
perform the following transpositions:

$$
\mathbf{A}^{Q_{A}} \mathbf{X}_{[2,-1]}^{Q_{1}} \mathbf{X}_{[-1,2]}^{Q_{2}} \mathbf{X}_{[1,1]}^{Q_{3}} \stackrel{P_{1} P_{2} P_{3} P_{3} P_{2} P_{1}}{\longrightarrow} \mathbf{A}^{Q_{A}-Q_{1}+2 Q_{2}+Q_{3}} \mathbf{X}_{[3,-1]}^{Q_{1}+Q_{A}} \mathbf{X}_{[-3,2]}^{Q_{2}+Q_{A}} \mathbf{X}_{[0,1]}^{Q_{3}-Q_{A}}
$$

This transposition consists of moving the A-brane around the other 7-branes once in the counter clockwise direction. The linear transformation $g$ induced on the charges is given by

$$
\left(\begin{array}{l}
Q_{A} \\
Q_{1} \\
Q_{2} \\
Q_{3}
\end{array}\right) \mapsto\left(\begin{array}{rrrr}
1 & -1 & 2 & 1 \\
1 & 1 & 0 & 0 \\
1 & 0 & 1 & 0 \\
-1 & 0 & 0 & 1
\end{array}\right)\left(\begin{array}{l}
Q_{A} \\
Q_{1} \\
Q_{2} \\
Q_{3}
\end{array}\right)
$$

The effect on the charges of moving the A-brane around the other 7-branes three times is given by the linear transformation $g^{3}$. We can restore the original configuration by transposition $P_{2} P_{3}$,

$$
\mathbf{A}^{Q_{A}} \mathbf{X}_{[2,-1]}^{Q_{1}} \mathbf{X}_{[-1,2]}^{Q_{2}} \mathbf{X}_{[1,1]}^{Q_{3}} \stackrel{\left(P_{1} P_{2} P_{3} P_{3} P_{2} P_{1}\right)^{3}}{\longrightarrow} \mathbf{A}^{Q_{A}^{\prime \prime \prime}} \mathbf{X}_{[5,-1]}^{Q_{1 \prime \prime}^{\prime \prime \prime}} \mathbf{X}_{[-7,2]}^{Q_{2 \prime \prime}^{\prime \prime}} \mathbf{X}_{[-2,1]}^{Q_{3 \prime}^{\prime \prime \prime}} \stackrel{P_{2} P_{3}}{\longrightarrow} \mathbf{A}^{\widehat{Q}_{A}} \mathbf{X}_{[2,-1]}^{\widehat{Q}_{1}} \mathbf{X}_{[-1,2]}^{\widehat{Q}_{2}} \mathbf{X}_{[1,1]}^{\widehat{Q}_{3}}
$$

The transformed charges $\widehat{Q}$ are

$$
\widehat{Q}_{A}=Q_{A}^{\prime \prime \prime}, \widehat{Q}_{1}=Q_{3}^{\prime \prime \prime}+3 Q_{2}^{\prime \prime \prime}-3 Q_{1}^{\prime \prime \prime}, \widehat{Q}_{2}=Q_{1}^{\prime \prime \prime}, \widehat{Q}_{3}=-Q_{2}^{\prime \prime \prime},
$$

where $\vec{Q}^{\prime \prime \prime}$ are obtained from $\vec{Q}$ by the linear transformation $g^{3}$. Using (3.9) we get

$$
\begin{aligned}
& \widehat{Q}_{A}=Q_{A}-3 Q_{1}+6 Q_{2}+3 Q_{3}, \\
& \widehat{Q}_{1}=3 Q_{A}+3 Q_{2}+2 Q_{3}, \\
& \widehat{Q}_{2}=3 Q_{A}-2 Q_{1}+6 Q_{2}+3 Q_{3}, \\
& \widehat{Q}_{3}=-3 Q_{A}+3 Q_{1}-7 Q_{2}-3 Q_{3} .
\end{aligned}
$$

Thus we see that $\left(\mathbb{1}, P_{2} P_{3}\left(P_{1} P_{2} P_{3} P_{3} P_{2} P_{1}\right)^{3}\right) \in \mathcal{H}(w)$ has a nontrivial action on the charges. Since there are no real root junctions with support on this configuration this element of $\mathcal{H}(w)$ is not of Weyl type. The motion of the $\mathbf{A}$-brane around the other three branes has the effect equivalent to the action of a global $S L(2, \mathbf{Z})$ transformation by $T^{-3}$. We will discuss this action in more detail in section 6 .

\section{Duality groups and Dynkin graph automorphisms}

The results of the previous section allow us to find and characterize the duality group $\mathcal{D}(w)$ of a brane configuration $w$ with monodromy $K(w)$. Since crossing transformations cannot change the monodromy $K(w)$ of the configuration, any element of the duality group must 
leave the monodromy invariant and therefore is contained in $\operatorname{Stab}(K)$, the stabilizer of $K$ in $S L(2, \mathbf{Z})\left(g \in \operatorname{Stab}(K) \Leftrightarrow g K g^{-1}=K\right)$

$$
\mathcal{D}(w) \subseteq \operatorname{Stab}(K(w)) .
$$

The duality group $\mathcal{D}(w)$ of the configuration will be the subgroup of $\operatorname{Stab}(K)$ that leaves the configuration invariant in the sense discussed in the previous section (see (2.8)). In any concrete case it is relatively straightforward to determine the group $\operatorname{Stab}(K)$. Then one must select $\mathcal{D}$ by finding the subgroup of $\operatorname{Stab}(K)$ for which there exist crossing transformations that restore the configuration.

We have seen that $K \in \mathcal{D}$ (Proposition, sect. 2.1) and therefore $\{K\}$, the group generated by $K$, is a subgroup of the duality group $\mathcal{D}$. Since $\{K\}$ is a normal subgroup of $\operatorname{Stab}(K)$, it is also a normal subgroup of $\mathcal{D}$. We are thus led to define the quotient group

$$
\overline{\mathcal{D}} \equiv \mathcal{D} /\{K\}
$$

referred to as the reduced duality group, that will play an important role in the computations. Another general fact discussed before is that the transformation $-\mathbb{1} \in S L(2, \mathbf{Z})$, clearly contained in $\operatorname{Stab}(K)$ is also an element in $\mathcal{D}$.

Consider now the action of an element $(g, b) \in \mathcal{S}(w)$ of the symmetry group of the brane configuration. The set of transpositions $b$ that restore the original configuration via $(g, b) w=$ $w$ will shuffle the invariant charges of junctions and therefore this symmetry maps junctions to (typically) different junctions. One nevertheless gets an automorphism of the junction lattice $\Lambda_{\mathbf{J}}$; namely for any two junctions $\mathbf{J}_{\mathbf{1}}$ and $\mathbf{J}_{\mathbf{2}}$ mapping to $\mathbf{J}_{1}^{\prime}$ and $\mathbf{J}_{2}^{\prime}$ one has $\mathbf{J}_{1} \cdot \mathbf{J}_{2}=\mathbf{J}_{1}^{\prime} \cdot \mathbf{J}_{2}^{\prime}$. We therefore have a map

$$
\mathcal{S} \rightarrow \operatorname{Aut}\left(\Lambda_{\mathbf{J}}\right)
$$

from the symmetry group of the brane configuration to the group of automorphisms of the junction lattice.

Let us focus for the moment on junctions representing roots of the Lie algebra associated to the brane configuration. In fact, more generally, consider junctions associated to elements in the root lattice $Q$ of the Lie algebra. Such junctions, as discussed in length in earlier papers [6] have zero asymptotic charges. It follows that symmetry transformations in $\mathcal{S}$ will map these junctions among themselves. In addition, for such junctions $\mathbf{J}_{1} \cdot \mathbf{J}_{2}=-\lambda_{1} \cdot \lambda_{2}$, where $\lambda_{1}, \lambda_{2} \in Q$ are the associated elements in the root lattice. Since duality elements map to automorphisms of the junction lattice, by restricting to junctions associated to $Q$ duality elements map to automorphisms of the root lattice $Q$. We therefore have:

$$
\mathcal{S} \rightarrow \operatorname{Aut}(Q)
$$


On the other hand there is no canonical map $\mathcal{D} \rightarrow \operatorname{Aut}\left(\Lambda_{\mathbf{J}}\right)$, nor there is a canonical map $\mathcal{D} \rightarrow$ $\operatorname{Aut}(Q)$ from the duality group of the configuration. This is so because for duality elements, the compensating crossing transformation in $\mathcal{H}(w)$ used to restore the brane configuration is not uniquely defined. As discussed in section 3, a brane configuration typically admits crossing transformations $\widehat{b}$ that leave it invariant. In fact, any Weyl reflection of the root lattice is generated on junctions by a crossing transformation $\widehat{b}$ that leaves the configuration invariant. In the language of (2.8) the ambiguous action of a duality transformation $g$ on junctions arises because the computation of such action requires the choice of some $b$ such that $(g, b) w=w$. But $b$ is ambiguous, if $b$ satisfies this equation, $b \widehat{b}$ does as well.

If a configuration $w$ has the property that $\mathcal{H}(w)=\mathcal{H}_{W}(w)$, namely, every invariant transposition is of Weyl type, then the map

$$
\mathcal{D} \rightarrow \operatorname{Aut}(Q) / W .
$$

is well defined. This is the case because all invariant transpositions map to the Weyl group. Since the duality elements $\{K\}$ always map to Weyl group we also have the well defined map

$$
\overline{\mathcal{D}} \rightarrow \operatorname{Aut}(Q) / W .
$$

In the above homomorphisms the quotient group to the right is well defined since $W$ is a normal subgroup of $\operatorname{Aut}(Q)$ (for this and other facts quoted below see, [20). This quotient, for algebras of finite type, is simply the (graph) automorphism group $\Gamma$ of the Dynkin diagram (and the \pm above is not necessary). For infinite Kac-Moody algebras the above quotient includes, in addition to the graph automorphism $\Gamma$, the generator $(-1)$ which changes the sign of every vector in the root lattice, and this is never a Weyl transformation. For finite algebras the transformation $(-1)$ of $Q$ is many times a Weyl transformation. From the list of finite algebras we consider, $(-1)$ is not a Weyl element for the $A_{N}$ series and for $E_{6}$. In such cases, $(-1)$ is equivalent, up to Weyl transformations, to a graph automorphism. Since the element $-\mathbb{1} \in \mathcal{D} \subseteq S L(2, \mathbf{Z})$ precisely acts as $(-1)$ on $Q$ this shows that this transformation is nontrivial (i.e. not Weyl) for the $A_{N}$ series, for $E_{6}$ and for affine algebras.

In the next section we will show that $\mathcal{H}(w)=\mathcal{H}_{W}(w)$ for 7 -brane configurations realizing finite algebras. This condition, however, may not satisfied for configurations realizing affine algebras, as we illustrated in section 3.2. Therefore in these cases (4.5) and (4.6) are not well defined since there is no unique choice of invariant transposition and different choices can induce non-Weyl action on the roots.

Our strategy in this case would be to define the maps $\mathcal{D} \rightarrow \pm \Gamma$ and $\overline{\mathcal{D}} \rightarrow \pm \Gamma$ with respect to a fixed set of invariant transpositions that can undo the effect of an $S L(2, \mathbf{Z})$ transformation. The map is thus dependent on the choice of transpositions. An invariant characterization will require better understanding of the structure of $\mathcal{H}(w)$. 
When defined, our interest is in the homomorphisms $\mathcal{D} \rightarrow \pm \Gamma$ and $\overline{\mathcal{D}} \rightarrow \pm \Gamma$, the latter capturing the interplay of duality transformations with Lie algebraic data. Dualities in $\mathcal{D}$ that map to nontrivial elements of $\Gamma$ relate junctions appearing in different representations or junctions appearing as vectors in $Q$ that are not related by Weyl transformations. For the finite algebras, we shall find cases when the map $\phi: \overline{\mathcal{D}} \rightarrow \pm \Gamma$ is an isomorphism (for $E_{6}$, for example), and cases when it is onto but not one to one ( $D_{4}$, for example).

We can readily find the implications for weight vectors in the case of configurations leading to finite algebras. In this situation $\mathbf{J}_{1} \cdot \mathbf{J}_{2}=-\lambda_{1} \cdot \lambda_{2}+f\left(p_{1}, q_{1} ; p_{2}, q_{2}\right)$, where $f$ is a quadratic form determined solely by the monodromy $K[6]$. Since duality transformations preserve $K$ the automorphisms of the junction lattice arising from dualities give automorphisms of the weight lattice $\Lambda$. On the other hand for finite algebras $\operatorname{Aut}(Q)=\operatorname{Aut}(\Lambda)$ since $\Lambda=Q^{*}$. It thus follows that the homomorphism $\mathcal{D} \rightarrow \pm \Gamma$ carries information on how representations in different conjugacy classes are mapped into each other by duality transformations. We leave the question of duality action on junctions corresponding general affine weight vectors open.

\section{Duality groups for finite-type configurations}

In this section we calculate duality groups $\mathcal{D}$ and give the homomorphisms to the corresponding Dynkin-graph automorphism groups $\Gamma$. These homomorphisms are characterized by $\phi: \overline{\mathcal{D}} \rightarrow \Gamma$, as discussed before. This section focuses on brane configurations of elliptic and parabolic monodromies realizing finite Lie algebras. The case of finite algebras is relatively simple to analyze. The 7-brane configurations realizing finite algebras have the property that $\mathcal{H}(w)=\mathcal{H}_{W}(w)$ i.e, all invariant transpositions are of Weyl type. To prove this consider the action of a invariant transposition on the weight vector of a junction with support on the 7-brane configuration. If the transposition is not of Weyl type it will induce an outer automorphism on the weight vector. It was shown in [5] that for 7-brane configurations realizing finite algebras the conjugacy class of a weight vector corresponding to a junction is determined by the asymptotic charge of the junction. Since a transposition cannot change the asymptotic charge of a junction, it cannot change the conjugacy class of the corresponding weight vector. Therefore the action of the transposition on the weight vector cannot be an outer automorphism and hence must be a Weyl transformation. This simplification implies that the homomorphism $\overline{\mathcal{D}} \mapsto \Gamma$ is well defined for these cases. In table 1 we list the relation between the conjugacy classes and the asymptotic charge for various 7-brane configurations realizing finite algebras. In the case of $E_{8}$ since there are no outer automorphisms therefore every transpositions is trivially of Weyl type. This is consistent with the fact that there is a single conjugacy class for $E_{8}$. 


\begin{tabular}{||c|l|c|c||}
\hline $\mathcal{G}$ & $w$ & $\mathcal{C}$ & constraint \\
\hline$A_{N}$ & $\mathbf{A}^{\mathbf{N}+\mathbf{1}}, \mathbf{A}^{\mathbf{N}+\mathbf{1}} \mathbf{C}$ & $\mathbf{Z}_{N}$ & $p(\bmod N)$ \\
\hline$D_{N}$ & $\mathbf{A}^{\mathbf{N}} \mathbf{B C}$ & $\mathbf{Z}_{2} \times \mathbf{Z}_{2}, N=$ even & $p-q(\bmod 2), q(\bmod 2)$ \\
\hline & & $\mathbf{Z}_{4}, N=$ odd & $2 p-q(\bmod 4)$ \\
\hline$E_{6}$ & $\mathbf{A}^{5} \mathbf{B C C}$ & $\mathbf{Z}_{3}$ & $p(\bmod 3)$ \\
\hline$E_{7}$ & $\mathbf{A}^{\mathbf{6}} \mathbf{B C C}$ & $\mathbf{Z}_{2}$ & $p+q(\bmod 2)$ \\
\hline
\end{tabular}

Table 1: Conjugacy classes of finite algebras, their group structure $\mathcal{C}$, and the asymptotic charges of junctions representing such conjugacy classes.

Throughout this and the next section we denote by $\{\cdots\}$ a group generated by the elements indicated by dots. In addition $\{\cdots \mid \cdots\}$ will denote the group generated by the elements to the left of the vertical bar, modulo the relations to the right of the bar.

\section{1 $\quad \mathrm{A}_{\mathrm{N}}$ configuration: $\mathrm{A}^{\mathrm{N}+1}$}

This configuration is built from $(N+1)[1,0]$ branes. The monodromy is $K=T^{-N-1}$ and $\operatorname{Stab}(K)=\{-\mathbb{1}, T\}$. Since $T$ preserves the charges of the 7-branes, it belongs to the duality group just like $-\mathbb{1} \in \mathcal{D}$. Therefore $\mathcal{D}\left(\mathbf{A}_{\mathbf{N}}\right)=\operatorname{Stab}(K)=\{-\mathbb{1}, T\}=\mathbf{Z}_{2} \times \mathbf{Z}$, and $\overline{\mathcal{D}}\left(\mathbf{A}_{\mathbf{N}}\right)=\left\{-\mathbb{1}, T \mid T^{N+1}=\mathbb{1}\right\}=\mathbf{Z}_{2} \times \mathbf{Z}_{N+1}$.

On the other hand $\Gamma\left(A_{N}\right)=\mathbf{Z}_{2}$ for $N \geq 2$ and is generated by the transformation $\mathcal{O}$ : $\left(a_{1}, a_{2}, \cdots, a_{n}\right) \mapsto\left(a_{n}, a_{n-1}, \cdots, a_{1}\right)$ of the Dynkin labels. Since $T$ does not affect the invariant charges it leaves all Dynkin labels unchanged and therefore $\phi(T)=\mathbb{1} \in \Gamma\left(A_{N}\right)$. The transformation $-\mathbb{1}$, however, changes the sign of all invariant charges and therefore of all the Dynkin labels

$$
-\mathbb{1}:\left(a_{1}, a_{2}, \cdots, a_{n}\right) \mapsto\left(-a_{1},-a_{2}, \cdots,-a_{n}\right) .
$$

The Weyl transformation $W$ which corresponds to rotating the 7-brane configuration by half a full turn can be seen to map

$$
W:\left(a_{1}, a_{2}, \cdots, a_{n}\right) \mapsto\left(-a_{n},-a_{n-1}, \cdots,-a_{1}\right) .
$$

We now recognize that the action of $-\mathbb{1} \in S L(2, \mathbf{Z})$ on the Dynkin labels is given by the composition of $\mathcal{O}$ and $W$. Therefore the homomorphism $\phi$ from $\mathcal{D}$ to $\Gamma\left(A_{N}\right)$ is defined as

$$
\begin{aligned}
\phi(T) & =+1 \in \Gamma\left(A_{N}\right) \\
\phi(-\mathbb{1}) & =\mathcal{O} \in \Gamma\left(A_{N}\right) .
\end{aligned}
$$




\begin{tabular}{|c|l|c|c|c|c|}
\hline$w$ & $K(w)$ & $\mathcal{D}(w)$ & $\overline{\mathcal{D}}(w)$ & $\mathcal{G}$ & $\Gamma(\mathcal{G})$ \\
\hline $\mathbf{A}_{\mathbf{1}}$ & $T^{-2}$ & $\{-\mathbb{1}, T\}$ & $\mathbf{Z}_{2} \times \mathbf{Z}_{2}$ & $A_{1}$ & 1 \\
\hline $\mathbf{A}_{\mathbf{N} \geq \mathbf{2}}$ & $T^{-N-1}$ & $\{-\mathbb{1}, T\}$ & $\mathbf{Z}_{2} \times \mathbf{Z}_{N+1}$ & $A_{N}$ & $\mathbf{Z}_{2}$ \\
\hline
\end{tabular}

Table 2: Duality groups and graph automorphisms for $\mathbf{A}_{\mathbf{N}}$ configurations.

In case of $\mathbf{A}_{\mathbf{1}}$ the computation of the duality group is identical and therefore $\overline{\mathcal{D}}\left(\mathbf{A}_{\mathbf{1}}\right)=$ $\mathbf{Z}_{2} \times \mathbf{Z}_{2}$. On the other hand here $\Gamma\left(A_{1}\right)=1$ and the $-\mathbb{1}$ transformation is simply a Weyl transformation, thus the homomorphism $\phi$ is trivial.

\section{$5.2 \quad \mathrm{H}_{\mathrm{N}}$ configurations: $\mathrm{A}^{\mathrm{N}+1} \mathrm{C}$}

Since $\mathbf{H}_{\mathbf{3}}=\mathbf{D}_{\mathbf{3}}$, and $\mathbf{H}_{\mathbf{N} \geq \mathbf{4}}$ have hyperbolic monodromies, we need only focus on the configurations $\mathbf{H}_{\mathbf{0}}, \mathbf{H}_{\mathbf{1}}$ and $\mathbf{H}_{\mathbf{2}}$.

- $\mathbf{H}_{\mathbf{0}}$ : The monodromy $K\left(\mathbf{H}_{\mathbf{0}}\right) \sim(S T)^{-1}$ and $\operatorname{Stab}(K)=\left\{(S T)^{-1}\right\}(-\mathbb{1} \in \operatorname{Stab}(K)$ since $\left.(S T)^{3}=-\mathbb{1}\right)$. Since $K \in \mathcal{D}, \mathcal{D}\left(\mathbf{H}_{\mathbf{0}}\right)=\operatorname{Stab}(K)=\mathbf{Z}_{6}$, and $\overline{\mathcal{D}}\left(\mathbf{H}_{\mathbf{0}}\right)=1$. This configuration supports no junctions without asymptotic charges so there is no $\mathcal{G}$ associated to it.

- $\mathbf{H}_{\mathbf{1}}$ : Here $K\left(\mathbf{H}_{\mathbf{1}}\right) \sim S^{-1}$ and $\operatorname{Stab}(K)=\{S\}$. Since $K \in \mathcal{D}, \mathcal{D}\left(\mathbf{H}_{\mathbf{1}}\right)=\operatorname{Stab}(K)=\mathbf{Z}_{4}$ and $\overline{\mathcal{D}}\left(\mathbf{H}_{\mathbf{1}}\right)=1$. Since $\Gamma\left(A_{1}\right)=1, \phi$ is the trivial isomorphism.

- $\mathbf{H}_{\mathbf{2}}$ : In this case $K\left(\mathbf{H}_{\mathbf{2}}\right) \sim-S T$ and $\operatorname{Stab}(K)=\{S T\}$. Since $(S T)^{3}=-\mathbb{1}, \mathcal{D}\left(\mathbf{H}_{\mathbf{2}}\right)=$ $\operatorname{Stab}(K)=\mathbf{Z}_{6}$ and $\overline{\mathcal{D}}\left(\mathbf{H}_{\mathbf{2}}\right)=\mathbf{Z}_{2}$. Here $\Gamma\left(A_{2}\right)=\mathbf{Z}_{2}$, and it follows from (5.1) and (5.2) that the homomorphism $\phi$ from $\overline{\mathcal{D}}\left(\mathbf{H}_{\mathbf{2}}\right)$ to $\Gamma\left(A_{2}\right)$ is given by

$$
\begin{aligned}
\phi(\mathbb{1}) & =+1 \in \Gamma\left(A_{2}\right), \\
\phi(-\mathbb{1}) & =\mathcal{O} \in \Gamma\left(A_{2}\right),
\end{aligned}
$$

where $\mathcal{O}\left(a_{1}, a_{2}\right)=\left(a_{2}, a_{1}\right)$.

\section{$5.3 \quad \mathrm{D}_{\mathrm{N}}$ configurations: $\mathrm{A}^{\mathrm{N}} \mathrm{BC}$}

We begin with some general remarks applicable whenever $N \neq 0$. We will show that $T \in \mathcal{D}$. Indeed a $T$ transformation can be undone by taking the rightmost $\mathbf{A}$ brane on a round trip 


\begin{tabular}{|c|c|c|c|c|c|}
\hline$w$ & $K(w)$ & $\mathcal{D}(w)$ & $\overline{\mathcal{D}}(w)$ & $\mathcal{G}$ & $\Gamma(\mathcal{G})$ \\
\hline $\mathbf{H}_{\mathbf{0}}$ & $(S T)^{-1}$ & $\{S T\}=\mathbf{Z}_{6}$ & 1 & -- & -- \\
\hline $\mathbf{H}_{\mathbf{1}}$ & $S^{-1}$ & $\{S\}=\mathbf{Z}_{4}$ & 1 & $A_{1}$ & 1 \\
\hline $\mathbf{H}_{\mathbf{2}}$ & $-(S T)$ & $\{S T\}=\mathbf{Z}_{6}$ & $\mathbf{Z}_{2}$ & $A_{2}$ & $\mathbf{Z}_{2}$ \\
\hline
\end{tabular}

Table 3: Duality groups and graph automorphisms for $\mathbf{H}_{\mathbf{N}}$ configurations.

encircling $\mathbf{B C}$ branes:

$$
\mathbf{A}^{\mathbf{N}-\mathbf{1}} \mathbf{A} \mathbf{B C} \stackrel{T}{\longrightarrow} \mathbf{A}^{\mathbf{N}-\mathbf{1}} \mathbf{A ~ X}_{[\mathbf{0},-\mathbf{1}]} \mathbf{X}_{[\mathbf{2}, \mathbf{1}]} \stackrel{P_{N} P_{N+1} P_{N+1} P_{N}}{\longrightarrow} \mathbf{A}^{\mathbf{N}-\mathbf{1}} \mathbf{A} \mathbf{B C} .
$$

In doing this operation the invariant charges on the branes, denoted as $Q_{1}, \cdots Q_{N-1}$, for the inert $\mathbf{A}$ branes, $Q_{N}$ for the rightmost $\mathbf{A}$ brane, and $Q_{B}, Q_{C}$, transform as

$$
\begin{aligned}
Q_{i} & \stackrel{T}{\longrightarrow} Q_{i}, \quad i=1 \ldots N-1 \\
Q_{N} & \stackrel{T}{\longrightarrow}-Q_{N}-Q_{B}+Q_{C}, \\
Q_{B} & \stackrel{T}{\longrightarrow} Q_{B}+Q_{N}, \\
Q_{C} & \stackrel{T}{\longrightarrow} Q_{C}+Q_{N} .
\end{aligned}
$$

On the other hand, we know form eq.(6.27) of [5] how Dynkin labels are given in terms of invariant charges:

$$
\begin{aligned}
a_{i} & =Q_{i}-Q_{i+1}, \quad i=1 \ldots N-2 \\
a_{N-1} & =Q_{N-1}-Q_{N}, \\
a_{N} & =Q_{N-1}+Q_{N}+Q_{B}-Q_{C} .
\end{aligned}
$$

One immediately deduces from the last two equations the action of the duality $T$ on the Dynkin labels

$$
T:\left(a_{1}, \cdots, a_{N-1}, a_{N}\right) \mapsto\left(a_{1}, \cdots, a_{N}, a_{N-1}\right) .
$$

This exchange of the last two Dynkin labels is the familiar $\mathbf{Z}_{2}$ automorphism of the $D_{N}$ Dynkin graph.

- $\mathbf{D}_{\mathbf{0}}$ : The monodromy $K(\mathbf{B C})=-T^{4}$ and $\operatorname{Stab}(K)=\{-\mathbb{1}, T\}$. A junction of asymptotic charge $(p, q)$ on this configuration satisfies the condition $p+q \equiv 0(\bmod 2)$. After a transformation by $T^{k}$ if the branes can be brought back to the original ones by 
branch cut moves then the transformed asymptotic charge $\left(p^{\prime}, q^{\prime}\right)=(p+k q, q)$ must also satisfy the same condition. This implies that $k \equiv 0(\bmod 2)$. Indeed, a probe D3brane in this background realizes $\mathcal{N}=2$ pure SW-theory whose BPS spectrum is not invariant under $T$ transformation [6]. One can verify, however, that this configuration is invariant under transformation by $T^{2}$ :

$$
\mathrm{BC} \stackrel{T^{2}}{\longrightarrow} \mathbf{C X}_{[\mathbf{3}, \mathbf{1}]} \stackrel{P_{1}^{-1}}{\longrightarrow} \mathrm{BC}
$$

It then follows that $\mathcal{D}\left(\mathbf{D}_{\mathbf{0}}\right)=\left\{-\mathbb{1}, T^{2}\right\}$ and $\overline{\mathcal{D}}\left(\mathbf{D}_{\mathbf{0}}\right)=\left\{-T^{2} \mid T^{4}=-\mathbb{1}\right\}=\mathbf{Z}_{4}$. Since this configuration supports no junctions without asymptotic charges there is no $\mathcal{G}$ associated to it.

- $\mathbf{D}_{1}$ : Here $K\left(\mathbf{D}_{1}\right)=-T^{3}$ and $\operatorname{Stab}(K)=\{-\mathbb{1}, T\}$. Since we have an $\mathbf{A}$ brane $T \in \mathcal{D}$. Thus $\mathcal{D}\left(\mathbf{D}_{\mathbf{1}}\right)=\{-\mathbb{1}, T\}$ and $\overline{\mathcal{D}}\left(\mathbf{D}_{\mathbf{1}}\right)=\left\{T \mid T^{3}=-\mathbb{1}\right\}=\mathbf{Z}_{6}$. This configuration does not support any root, therefore there is no Dynkin diagram. Nevertheless, as is well known, it carries a $u(1)$ algebra, whose associated junction is the non-BPS junction $\overline{\mathbf{J}}=2 \mathbf{a}-\mathbf{b}-\mathbf{c}$ [14]. For an arbitrary junction $\mathbf{J}$ the corresponding $u(1)$ charge $Q^{*}$ is proportional to $\mathbf{J} \cdot \overrightarrow{\mathbf{J}} \sim 2 Q_{1}+Q_{B}-Q_{C}$. Both $-\mathbb{1}$ and $T$ are checked to take $Q^{*} \rightarrow-Q^{*}$.

- $\mathbf{D}_{\mathbf{2}}$ : This configuration has $K\left(\mathbf{D}_{\mathbf{2}}\right)=-T^{2}, \operatorname{Stab}(K)=\{-\mathbb{1}, T\}=\mathcal{D}\left(\mathbf{D}_{\mathbf{2}}\right)$. Therefore $\overline{\mathcal{D}}\left(\mathbf{D}_{\mathbf{2}}\right)=\left\{T \mid T^{2}=-\mathbb{1}\right\}=\mathbf{Z}_{4}$. The configuration supports two roots representing the $A_{1} \oplus A_{1}$ algebra, it corresponds to two disconnected Dynkin nodes, with Dynkin labels $a_{1}$ and $a_{2}$ correctly given by (5.7). The action of $T$ as given in (5.8) simply exchanges the two Dynkin labels. This is the non-trivial element of $\Gamma\left(A_{1} \oplus A_{1}\right)=\mathbf{Z}_{2}$. Therefore $\phi: \overline{\mathcal{D}}\left(\mathbf{D}_{\mathbf{2}}\right)=\mathbf{Z}_{4} \mapsto \mathbb{Z}_{2}$ via $\phi(T)=-1$.

- $\mathbf{D}_{\mathbf{3}}$ : Here $K\left(\mathbf{D}_{\mathbf{3}}\right)=-T, \operatorname{Stab}(K)=\{-\mathbb{1}, T\}=\mathcal{D}\left(\mathbf{D}_{\mathbf{3}}\right)$, and $\overline{\mathcal{D}}\left(\mathbf{D}_{\mathbf{3}}\right)=\{T \mid T=$ $-\mathbb{1}\}=\mathbb{Z}_{2}$. Also $T:\left(a_{1}, a_{2}, a_{3}\right)=\left(a_{1}, a_{3}, a_{2}\right)$, is the non-trivial element of $\Gamma\left(A_{3}\right)=\mathbb{Z}_{2}$ (the labeling of nodes follows the $D_{N}$ conventions; node number one is in the middle). Thus the homomorphism $\phi: \overline{\mathcal{D}}\left(\mathbf{D}_{\mathbf{3}}\right) \mapsto \Gamma\left(A_{3}\right)=\mathbf{Z}_{2}$ is the isomorphism $\phi(T)=-1$.

- $\mathbf{D}_{4}$ : In this case the monodromy is $-\mathbb{1}$ and therefore $\operatorname{Stab}(K)=S L(2, \mathbf{Z})$. Since invariance under $T$ has been already established, we show that $\mathcal{D}\left(\mathbf{D}_{\mathbf{4}}\right)=\operatorname{Stab}(K)=$ $S L(2, \mathbf{Z})$ by demonstrating the invariance of the configuration under $S$. Indeed,

$$
\mathbf{A}^{4} \mathbf{B C} \stackrel{S}{\longrightarrow}\left(\mathbf{X}_{[\mathbf{0}, \mathbf{1}]}\right)^{4} \mathbf{C B} \stackrel{\left(P_{5} P_{4} P_{3} P_{2} P_{1} P_{1} P_{2} P_{3} P_{4}\right)}{\longrightarrow} \mathbf{A}^{4} \mathbf{B C}
$$

It is possible to anticipate the action of $S$ on the $D_{4}$ Dynkin labels. Recall from [5] that the various conjugacy classes of $s o(8)$ are correlated with asymptotic $(p, q)$ charges $\bmod 2$. In particular in eq. (6.26) of [5] we see that $\boldsymbol{8}_{\mathbf{v}}$ and $\boldsymbol{8}_{\mathbf{s}}$ representations arise from $(1,0)$ and $(0,1)$ charges respectively $(\bmod 2)$, while $\boldsymbol{8}_{\mathbf{c}}$ arises from $(1,1)$. We see that 
$\bmod 2$, the action of $S$ on those asymptotic charges exchanges the ones corresponding to $\mathbf{8}_{\mathbf{v}}$ and $\mathbf{8}_{\mathbf{s}}$ while it leaves invariant that corresponding to $\mathbf{8}_{\mathbf{c}}$. In our conventions, $\mathbf{8}_{\mathbf{v}}$ and $\boldsymbol{8}_{\mathbf{s}}$ are associated to the first and third nodes of the Dynkin diagram, and therefore we expect $S$ to act as the graph automorphism $a_{1} \leftrightarrow a_{3}$.

The transformations in (5.10) imply that under $S$, the invariant charges transform as:

$$
\begin{aligned}
& Q_{i} \stackrel{S}{\longrightarrow} Q_{B}-Q_{i}+\sum Q_{k} \quad i=1 \ldots 4 \\
& Q_{B} \stackrel{S}{\longrightarrow}-Q_{C}-2 Q_{B}-2 \sum Q_{k} \\
& Q_{C} \stackrel{S}{\longrightarrow}-Q_{B}-\sum Q_{k} .
\end{aligned}
$$

The resulting action on the Dynkin labels (5.7) is given by

$$
S:\left(a_{1}, a_{2}, a_{3}, a_{4}\right) \mapsto\left(-a_{1},-a_{2},-a_{3}, a_{1}+2 a_{2}+a_{3}+a_{4}\right) .
$$

A little calculation shows that $S$ is a composition of Weyl reflections and the expected graph automorphism:

$$
S=\mathcal{O} W_{\alpha_{1}+\alpha_{2}+\alpha_{3}} W_{\alpha_{2}}, \quad \mathcal{O}\left(a_{1}, a_{2}, a_{3}, a_{4}\right) \equiv\left(a_{3}, a_{2}, a_{1}, a_{4}\right) .
$$

- $\mathbf{D}_{\mathbf{N} \geq \mathbf{5}}$ : Here $K\left(\mathbf{D}_{\mathbf{N}}\right)=-T^{4-N}$ and $\operatorname{Stab}(K)=\{-\mathbb{1}, T\}=\mathcal{D}\left(\mathbf{D}_{\mathbf{N}}\right)$. Thus $\overline{\mathcal{D}}\left(\mathbf{D}_{\mathbf{N}}\right)=$ $\left\{T \mid T^{N-4}=-\mathbb{1}\right\}=\mathbf{Z}_{2(N-4)}$. Under $T$, using (5.8), we have $\phi: \overline{\mathcal{D}}\left(\mathbf{D}_{\mathbf{N}}\right) \mapsto \Gamma\left(D_{N}\right)=$ $\mathbb{Z}_{2}$ is fixed by $\phi(T)=-1$. Since we have a homomorphism, $\phi(-\mathbb{1})=\phi\left(T^{N-4}\right)=$ $[\phi(T)]^{N-4}=(-1)^{N-4}=(-1)^{N}$. Thus for $\mathbf{D}_{N}$ with $N$ even, the transformation $-\mathbb{1}$ maps to a Weyl transformation, while for $N$ odd, the transformation $-\mathbb{1}$ is equivalent to the nontrivial graph automorphism up to a Weyl transformation. This is as expected; a change of sign of all Dynkin labels in the $D_{N}$ algebras is a Weyl transformation only for $N$ even (Ref. [21], sect. 13).

\section{4 $\mathrm{E}_{\mathrm{N}}$ configuration: $\mathrm{A}^{\mathrm{N}-1} \mathrm{BCC}$}

- $\mathbf{E}_{\mathbf{6}}$ : Here $K\left(\mathbf{E}_{\mathbf{6}}\right) \sim-(S T)^{-1}$ and $\operatorname{Stab}(K)=\{-\mathbb{1}, K\}=\mathbf{Z}_{6}=\mathcal{D}\left(\mathbf{E}_{\mathbf{6}}\right)$. Therefore $\overline{\mathcal{D}}\left(\mathbf{E}_{\mathbf{6}}\right)=\{-\mathbb{1}\}=\mathbf{Z}_{2}$. In addition, $\Gamma\left(E_{6}\right)=\mathbf{Z}_{2}$, and its non-trivial element, up to a Weyl transformation, changes the sign of all the Dynkin labels (maps representations to their conjugates). It follows that the homomorphism $\phi: \overline{\mathcal{D}}\left(\mathbf{E}_{\mathbf{6}}\right) \mapsto \mathbf{Z}_{2}$ is fixed by $\phi(-\mathbb{1})=-1$. This is an isomorphism. 


\begin{tabular}{|c|l|c|c|c|c|}
\hline$w$ & $K(w)$ & $\mathcal{D}(w)$ & $\overline{\mathcal{D}}(w)$ & $\mathcal{G}$ & $\Gamma(\mathcal{G})$ \\
\hline $\mathbf{D}_{\mathbf{0}}$ & $-T^{4}$ & $\left\{-\mathbb{1}, T^{2}\right\}$ & $\mathbf{Z}_{4}$ & -- & -- \\
\hline $\mathbf{D}_{\mathbf{1}}$ & $-T^{3}$ & $\{-\mathbb{1}, T\}$ & $\mathbf{Z}_{6}$ & $u(1)$ & -- \\
\hline $\mathbf{D}_{\mathbf{2}}$ & $-T^{2}$ & $\{-\mathbb{1}, T\}$ & $\mathbf{Z}_{4}$ & $A_{1} \oplus A_{1}$ & $\mathbf{Z}_{2}$ \\
\hline $\mathbf{D}_{\mathbf{3}}$ & $-T$ & $\{-\mathbb{1}, T\}$ & $\mathbf{Z}_{2}$ & $A_{3}$ & $\mathbf{Z}_{2}$ \\
\hline $\mathbf{D}_{\mathbf{4}}$ & $-\mathbb{1}$ & $S L(2, \mathbf{Z})$ & $P S L(2, \mathbf{Z})$ & $D_{4}$ & $S_{3}$ \\
\hline $\mathbf{D}_{\mathbf{4 + N} \geq \mathbf{5}}$ & $-T^{-N}$ & $\{-\mathbb{1}, T\}$ & $\mathbf{Z}_{2 N}$ & $D_{N}$ & $\mathbf{Z}_{2}$ \\
\hline
\end{tabular}

Table 4: Duality groups and graph automorphisms for $\mathbf{D}_{\mathbf{N}}$ configurations. $S_{3}$ is the permutation group of three objects.

\begin{tabular}{|c|c|c|c|c|c|}
\hline$w$ & $K(w)$ & $\mathcal{D}(w)$ & $\overline{\mathcal{D}}(w)$ & $\mathcal{G}$ & $\Gamma(\mathcal{G})$ \\
\hline $\mathbf{E}_{\mathbf{6}}$ & $-(S T)^{-1}$ & $\{S T\}=\mathbf{Z}_{6}$ & $\mathbf{Z}_{2}$ & $E_{6}$ & $\mathbf{Z}_{2}$ \\
\hline $\mathbf{E}_{\mathbf{7}}$ & $S$ & $\{S\}=\mathbf{Z}_{4}$ & 1 & $E_{7}$ & 1 \\
\hline $\mathbf{E}_{\mathbf{8}}$ & $S T$ & $\{S T\}=\mathbf{Z}_{6}$ & 1 & $E_{8}$ & 1 \\
\hline
\end{tabular}

Table 5: Duality groups and graph automorphisms for $\mathbf{E}_{\mathbf{N}}$ configurations.

- $\mathbf{E}_{\mathbf{7}}$ : Here $K\left(\mathbf{E}_{\mathbf{7}}\right) \sim S$ and $\operatorname{Stab}(K)=\{S\}=\mathbf{Z}_{4}=\mathcal{D}\left(\mathbf{E}_{\mathbf{7}}\right)$. It follows that $\overline{\mathcal{D}}\left(E_{7}\right)=\{\mathbb{1}\}$. Since $\Gamma\left(E_{7}\right)$ is also trivial the homomorphism $\phi$ is trivial. Dualities will preserve Weyl orbits, and therefore representations. While the duality $-\mathbb{1}=S^{2}$ changes the sign of all Dynkin labels, this is simply a Weyl transformation of $E_{7}$.

- $\mathbf{E}_{\mathbf{8}}$ : Here $K\left(\mathbf{E}_{\mathbf{8}}\right) \sim(S T)^{-1}$ and $\operatorname{Stab}(K)=\left\{(S T)^{-1}\right\}=\mathbf{Z}_{6}=\mathcal{D}\left(\mathbf{E}_{\mathbf{8}}\right)$. Just as in the case of $E_{7}$ we have $\overline{\mathcal{D}}\left(\mathbf{E}_{\mathbf{8}}\right)=\{\mathbb{1}\}, \Gamma\left(E_{8}\right)=1$ and a trivial homomorphism $\phi$.

\section{Duality groups for affine configurations}

In this section we will try to extend the result of previous section to the case of affine exceptional configurations $\widehat{\widetilde{\mathbf{E}}}_{0}, \widehat{\widetilde{\mathbf{E}}}_{1}$, and the series $\widehat{\mathbf{E}}_{N}$ for $1 \leq N \leq 8$. These configurations are more interesting because of their relation with del Pezzo surfaces [15] but at the same time more difficult to analyze since for these configurations $\mathcal{H}(w) \neq \mathcal{H}_{W}(w)$ i.e, not all transpositions are of Weyl type. This means that there are elements in $\mathcal{H}(w)$ whose action 
on roots may be outer automorphisms of the root lattice. Our strategy in this case will be to find the map $\overline{\mathcal{D}}(w) /\{-\mathbb{1}\} \mapsto \pm \Gamma$ for a fixed set of transformations used to undo the $S L(2, \mathbb{Z})$ transformations in $\mathcal{D}(w)$.

We begin with some general remarks applicable to the the affine exceptional brane configurations $\widehat{\mathbf{E}}_{\mathbf{N}}=\mathbf{A}^{\mathbf{N}-\mathbf{1}} \mathbf{B C B C}$ with $2 \leq N \leq 8$. All such configurations have at least one $\mathbf{A}$ brane. They have monodromy $K\left(\widehat{\mathbf{E}}_{\mathbf{N}}\right)=T^{9-\bar{N}}$, and one readily finds that $\operatorname{Stab}(K)=\{-\mathbb{1}, T\}$. We now show that $T \in \mathcal{D}$ for $n>1$ by an explicit calculation quite similar to that given in (5.5). We make the $\mathbf{A}$ brane do a counterclockwise round trip around the other branes:

$$
\mathbf{A B C B C} \stackrel{T}{\longrightarrow} \mathbf{A X}_{[\mathbf{0},-\mathbf{1}]} \mathbf{X}_{[\mathbf{2}, \mathbf{1}]} \mathbf{X}_{[\mathbf{0},-\mathbf{1}]} \mathbf{X}_{[\mathbf{2}, \mathbf{1}]} \stackrel{P_{1} P_{2} P_{3} P_{4} P_{4} P_{3} P_{2} P_{1}}{\longrightarrow} \mathbf{A B C B C}
$$

Let $\left\{Q_{A}, Q_{B}^{1}, Q_{C}^{1}, Q_{B}^{2}, Q_{C}^{2}\right\}$ denote the invariant charges on the branes. The transformed invariant charges are found to be

$$
\begin{aligned}
& Q_{A} \stackrel{T}{\longrightarrow} Q_{A}+q, \\
& Q_{B}^{1} \stackrel{T}{\longrightarrow} Q_{B}^{1}+Q_{A}, \\
& Q_{C}^{1} \stackrel{T}{\longrightarrow} Q_{C}^{1}+Q_{A}, \\
& Q_{B}^{2} \stackrel{T}{\longrightarrow} Q_{B}^{2}-Q_{A}, \\
& Q_{C}^{2} \stackrel{T}{\longrightarrow} Q_{C}^{2}-Q_{A} .
\end{aligned}
$$

Here, $q=Q_{C}^{1}+Q_{C}^{2}-Q_{B}^{1}-Q_{B}^{1}$ is the total $q$-charge of the junction $\mathbf{J}$. Since we are only interested in junctions that correspond to states in the root lattice, we set $q=0$. We see that the effect of the $T$ transformation on a junction of $\widehat{E}_{N}$ with zero $q$ charge is simply

$$
\mathbf{J} \mapsto \mathbf{J}+Q_{A}(\mathbf{J}) \boldsymbol{\delta} .
$$

Here, $Q_{A}(\mathbf{J})$ is the invariant charge on the $\mathbf{A}$-brane used to undo the effect of the $T$ transformation, and $\boldsymbol{\delta}=\mathbf{b}_{1}+\mathbf{b}_{2}-\mathbf{c}_{1}-\mathbf{c}_{2}$ [12]. Indeed, when there is more than one $\mathbf{A}$ brane, any of them can be used to undo the effect of $T$. We have therefore shown that

$$
\mathcal{D}\left(\widehat{\mathbf{E}}_{\mathbf{N}}\right)=\{-\mathbb{1}, T\}, \quad 2 \leq N \leq 8 .
$$

$\widehat{\mathbf{E}}_{\mathbf{9}}$ being the composition of two copies of $D_{4}$ has $\mathcal{D}\left(\widehat{\mathbf{E}}_{\mathbf{9}}\right)=S L(2, \mathbf{Z})$. In addition, since $K\left(\widehat{\mathbf{E}}_{\mathbf{N}}\right)=T^{9-N}$ we also have

$$
\overline{\mathcal{D}}\left(\widehat{\mathbf{E}}_{\mathbf{N}}\right)=\left\{-\mathbb{1}, T \mid T^{9-N}=1\right\}=\mathbf{Z}_{2} \times \mathbf{Z}_{9-N}, \quad 2 \leq N \leq 8 .
$$

The group $\operatorname{Aut}(Q) / W$ of an affine algebra, written as $\pm \Gamma$ in section 4 , is more precisely written as

$$
\operatorname{Aut}(Q) / W=\mathbf{Z}_{2} \times \Gamma
$$


where the element $(-1, e)$, with $e$ the identity in $\Gamma$, is the transformation $Q \rightarrow-Q$ reversing the sign of all the vectors in the root lattice, and thus reversing the sign of all Dynkin labels. An element of the form $(0, h \in \Gamma)$ simply acts by the graph automorphism $h$ of the Dynkin graph of the affine algebra. The duality $-\mathbb{1} \in \mathcal{D}\left(\widehat{\mathbf{E}}_{\mathbf{N}}\right)$ maps to $(1,0)$ in $\overline{\mathcal{D}}$ and then

$$
\phi:(1,0) \in \overline{\mathcal{D}}=\mathbf{Z}_{2} \times \mathbf{Z}_{9-N} \rightarrow(1, e) \in \mathbf{Z}_{2} \times \Gamma
$$

Our computations will require finding how $T \in \mathcal{D}$ acts. For this we note that it maps to $(0,1) \in \overline{\mathcal{D}}$. We will find that

$$
\phi:(0,1) \in \overline{\mathcal{D}}=\mathbf{Z}_{2} \times \mathbf{Z}_{9-N} \rightarrow(0, h(T)) \in \mathbf{Z}_{2} \times \Gamma,
$$

where $h(T)$ is a graph automorphism. This map respects the product structure of the groups involved.

To simplify the formulae we also introduce the following notation for Weyl transformations,

$$
W_{i_{1}^{n_{1}} i_{2}^{n_{2} \ldots i_{k}} n_{k}} \equiv W_{n_{1} \alpha_{i_{1}}+n_{2} \alpha_{i_{2}}+\cdots n_{k} \alpha_{i_{k}}} .
$$

Let us now consider in detail the various configurations in the above series. We will show the brane configurations and indicate the simple root junctions. Then we select an $\mathbf{A}$ brane to undo the $T$ duality and use equation (6.3) to find the action on the simple roots. The final step is writing this action as the composition of a Weyl transformation and the action arising from a Dynkin graph automorphism. The answer is the graph automorphism $h(T) \in \Gamma$ defined in equation (6.8).

- $\widehat{\mathbf{E}}_{\mathbf{2}}$ : The $T$ transformation acts trivially on the roots since the A-brane supports no root. Therefore

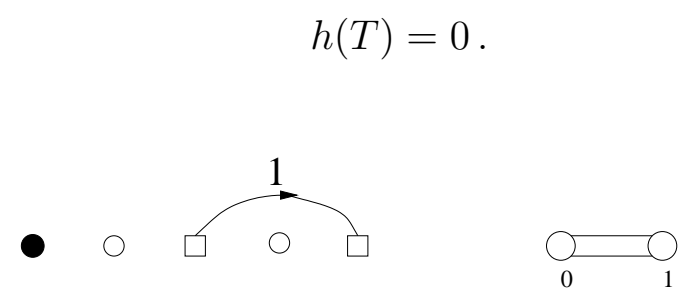

Figure 4: $\widehat{\mathbf{E}}_{2}$ brane configuration and $\widehat{A}_{1}$ Dynkin diagram.

- $\widehat{\mathbf{E}}_{3}$ : Using the rightmost $\mathbf{A}$ brane of the figure, $T$ acts on the simple roots as follows,

$$
T:\left(\alpha_{0}, \alpha_{1}, \alpha_{2} ; \beta_{0}, \beta_{1}\right) \rightarrow\left(\alpha_{0}-\delta, \alpha_{1}+\delta, \alpha_{2} ; \beta_{0}+\delta, \beta_{1}-\delta\right) .
$$


Here, $\left(\alpha_{0}, \alpha_{1}, \alpha_{2}\right)$ are the roots of $\widehat{A}_{2}$ and $\left(\beta_{0}, \beta_{1}\right)$ are the roots of $\widehat{A}_{1}$. It is easy to verify that acting on simple roots

$$
T=\left(W_{\alpha_{1}} W_{\alpha_{2}} \mathcal{O}, W_{\beta_{0}} \mathcal{O}^{\prime}\right)
$$

where the first and second terms indicate the action on the $\widehat{A}_{2}$ and $\widehat{A}_{1}$ roots respectively. The graph automorphisms $\mathcal{O}$ and $\mathcal{O}^{\prime}$ are

$$
\mathcal{O}\left(\alpha_{0}, \alpha_{1}, \alpha_{2}\right)=\left(\alpha_{2}, \alpha_{0}, \alpha_{1}\right), \quad \mathcal{O}^{\prime}\left(\beta_{0}, \beta_{1}\right)=\left(\beta_{1}, \beta_{0}\right) \text {. }
$$

These are elements of $\Gamma\left(\widehat{A}_{2} \oplus \widehat{A}_{1}\right)=\mathcal{D}_{6} \times \mathbf{Z}_{2}$, where $\mathcal{D}_{6}$ denotes the symmetry group of the triangle, here formed by the $\widehat{A}_{2}$ simple roots, with $\mathcal{O}$ the elementary rotation. In addition, $\mathcal{O}^{\prime}$ is the nontrivial element of $\mathbb{Z}_{2}$, representing the exchange of the two simple roots of $\widehat{A_{1}}$. In summary;

$$
h(T)=\left(\mathcal{O}, \mathcal{O}^{\prime}\right) \in \mathcal{D}_{6} \times \mathbf{Z}_{2}=\Gamma\left(\widehat{A}_{2} \oplus \widehat{A}_{1}\right) .
$$
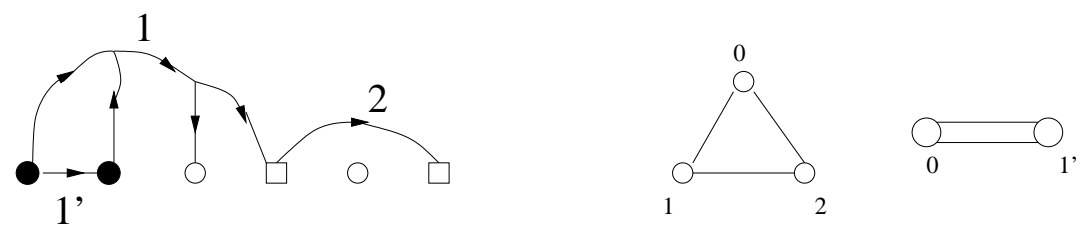

Figure 5: $\widehat{\mathbf{E}}_{3}$ brane configuration and $\widehat{A}_{2} \oplus \widehat{A}_{1}$ Dynkin diagram.

- $\widehat{\mathbf{E}}_{4}$ : Using the rightmost $\mathbf{A}$ brane the nontrivial action of $T$ on the roots is given by

$$
T:\left(\alpha_{2}, \alpha_{4}\right) \rightarrow\left(\alpha_{2}-\delta, \alpha_{4}+\delta\right)
$$

A calculation shows that

$$
T=\mathcal{O} W_{1} W_{2} W_{3} W_{0} W_{1} W_{2}, \quad \mathcal{O}\left(\alpha_{0}, \alpha_{1}, \alpha_{2}, \alpha_{3}, \alpha_{4}\right)=\left(\alpha_{3}, \alpha_{4}, \alpha_{0}, \alpha_{1}, \alpha_{2}\right)
$$

Here $\mathcal{O}$ implements the transformation $\omega^{3} \in \Gamma\left(\widehat{A}_{4}\right)=\mathcal{D}_{10}$, where $\omega$ is a cyclic minimal rotation of the pentagon. Note that $\omega^{3}$ is a generator for the $\mathbf{Z}_{5}$ subgroup of rotations of $\mathcal{D}_{10}$. Thus, in summary

$$
h(T)=\mathcal{O}=\omega^{3} \in \mathcal{D}_{10}
$$

Note that in $\overline{\mathcal{D}}, T^{5}=\mathbb{1}$, and this is consistent with the map to $\mathcal{D}_{10}$. 

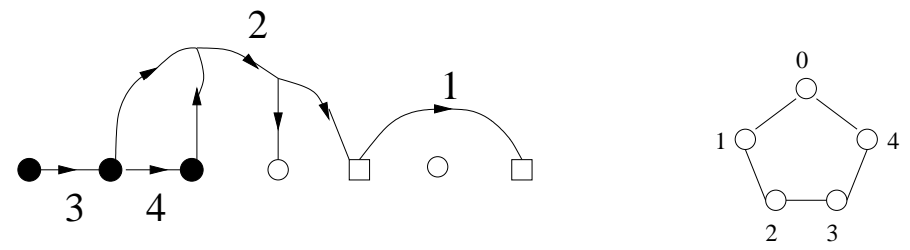

Figure 6: $\widehat{\mathbf{E}}_{4}$ brane configuration and $\widehat{A_{4}}$ Dynkin diagram.

- $\widehat{\mathbf{E}}_{5}$ : Using the rightmost $\mathbf{A}$ brane, the action of $T$ is:

$$
T:\left(\alpha_{0}, \alpha_{5}\right) \rightarrow\left(\alpha_{0}-\delta, \alpha_{5}+\delta\right)
$$

This time we find

$$
T=\mathcal{O} W_{2} W_{1234} W_{35}, \quad \mathcal{O}\left(\alpha_{0}, \alpha_{1}, \alpha_{2}, \alpha_{3}, \alpha_{4}, \alpha_{5}\right)=\left(\alpha_{4}, \alpha_{5}, \alpha_{3}, \alpha_{2}, \alpha_{1}, \alpha_{0}\right)
$$

Here $\mathcal{O} \in \Gamma\left(\widehat{D}_{5}\right)=\mathcal{D}_{8}$, is a generator for the $\mathbf{Z}_{4}$ subgroup of $\mathcal{D}_{8}$, consistent with $T^{4}=\mathbb{1}$ in $\overline{\mathcal{D}}$. In summary:

$$
h(T)=\mathcal{O} \in \Gamma\left(\widehat{D}_{5}\right)=\mathcal{D}_{8}
$$
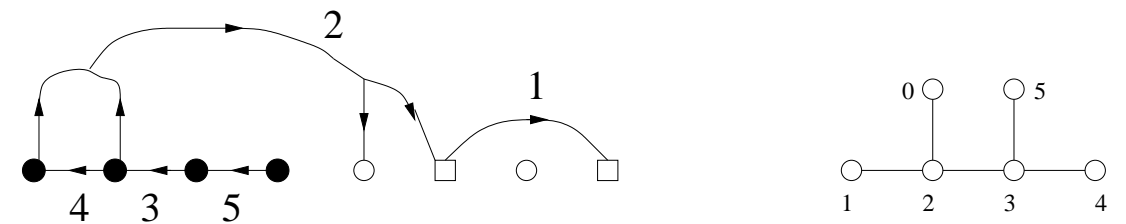

Figure 7: $\widehat{\mathbf{E}}_{5}$ brane configuration and $\widehat{D}_{5}$ Dynkin diagram.

- $\widehat{\mathbf{E}}_{\mathbf{6}}$ : Using the leftmost brane, one finds that $T$ induces the transformations

$$
T:\left(\alpha_{0}, \alpha_{5}\right) \rightarrow\left(\alpha_{0}-\delta, \alpha_{5}+\delta\right) .
$$

A calculation shows that

$$
T=\mathcal{O} W_{34} W_{1234} W_{1236} W_{23} W_{45},
$$

where $\mathcal{O} \in \Gamma\left(\widehat{E}_{6}\right)=S_{3}$ is the generator of the $\mathbb{Z}_{3}$ subgroup of $S_{3}$ performing the rigid minimal rotation of the Dynkin diagram:

$$
\mathcal{O}\left(\alpha_{0}, \alpha_{1}, \alpha_{2}, \alpha_{3}, \alpha_{4}, \alpha_{5}, \alpha_{6}\right)=\left(\alpha_{1}, \alpha_{5}, \alpha_{4}, \alpha_{3}, \alpha_{6}, \alpha_{0}, \alpha_{2}\right)
$$



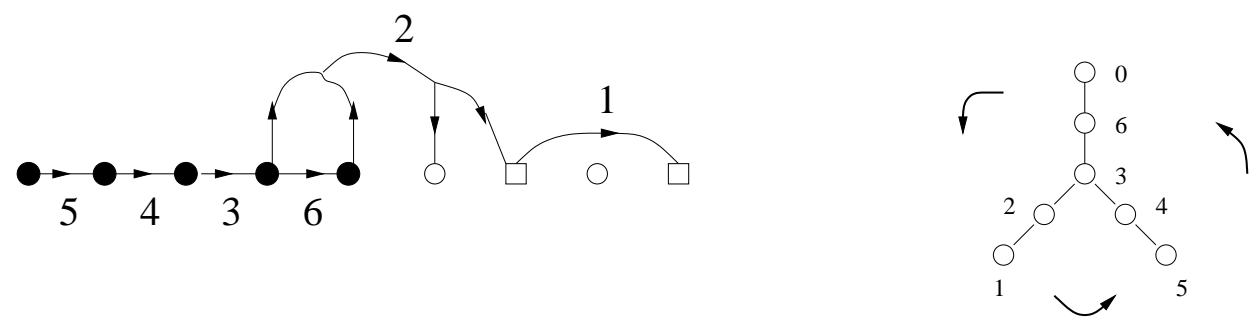

Figure 8: $\widehat{\mathbf{E}}_{6}$ brane configuration and $\widehat{E}_{6}$ Dynkin diagram.

This is compatible with $T^{3}=\mathbb{1}$ in $\overline{\mathcal{D}}$. In summary:

$$
h(T)=\mathcal{O} \in \Gamma\left(\widehat{E}_{6}\right)=S_{3} .
$$

- $\widehat{\mathbf{E}}_{\mathbf{7}}$ : Using the leftmost $\mathbf{A}$ brane, $T$ acts as

$$
\begin{aligned}
& T:\left(\alpha_{0}, \alpha_{1}\right) \rightarrow\left(\alpha_{0}-\delta, \alpha_{1}+\delta\right) \\
& T=\mathcal{O} W_{45} W_{45^{2} 67} W_{123^{2} 4567} W_{12^{2} 3^{2} 4^{2}{ }_{57}} W_{34}
\end{aligned}
$$

where $\mathcal{O} \in \Gamma\left(\widehat{E}_{7}\right)=\mathbf{Z}_{2}$ is the nontrivial generator of the graph automorphism, and exchanges the two long branches of the Dynkin diagram:

$$
\mathcal{O}\left(\alpha_{0}, \alpha_{1}, \alpha_{2}, \alpha_{3}, \alpha_{4}, \alpha_{5}, \alpha_{6}, \alpha_{7}\right)=\left(\alpha_{1}, \alpha_{0}, \alpha_{6}, \alpha_{5}, \alpha_{4}, \alpha_{3}, \alpha_{2}, \alpha_{7}\right)
$$

This is compatible with $T^{2}=\mathbb{1}$ in $\overline{\mathcal{D}}$. In summary:

$$
h(T)=\mathcal{O} \in \Gamma\left(\widehat{E}_{7}\right)=\mathbf{Z}_{2} .
$$
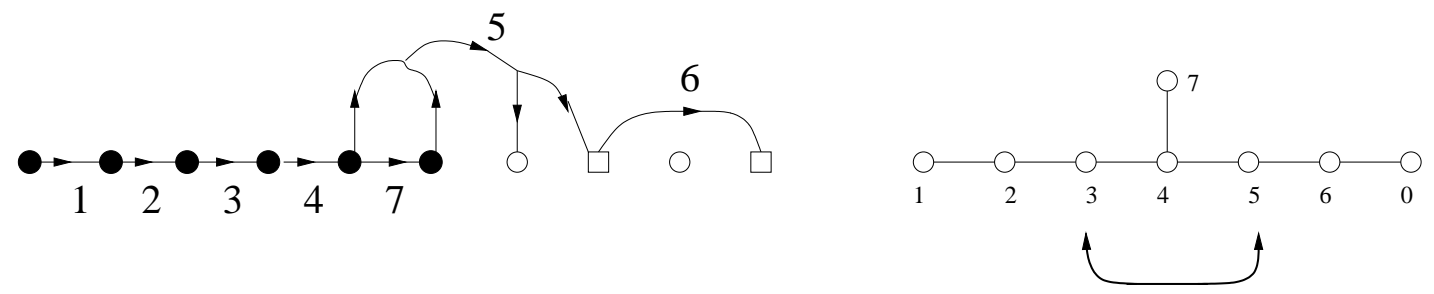

Figure 9: $\widehat{\mathbf{E}}_{7}$ brane configuration and $\widehat{E}_{7}$ Dynkin diagram.

- $\widehat{\mathbf{E}}_{\mathbf{8}}$ : Using the leftmost $\mathbf{A}$ brane the $T$ action is:

$$
T:\left(\alpha_{0}, \alpha_{1}\right) \mapsto\left(\alpha_{0}-2 \delta, \alpha_{1}+\delta\right)
$$


Since $\Gamma\left(\widehat{E}_{8}\right)=1$, the above ought to be a pure Weyl transformation. Indeed,

$$
\begin{array}{r}
T=W_{0} W_{123456} W_{123458} W_{34567} W_{2345^{2} 678} W_{6} W_{345^{2} 678} W_{234568} \\
\cdot W_{56} W_{1234567} W_{12} W_{12345678} W_{34568} W_{12345^{2} 68}
\end{array}
$$

Therefore $h(T)=e$ is the identity element in the trivial group $\Gamma\left(\widehat{E}_{8}\right)=1$. Indeed $T=\mathbb{1}$ in $\mathcal{D}$ as well.
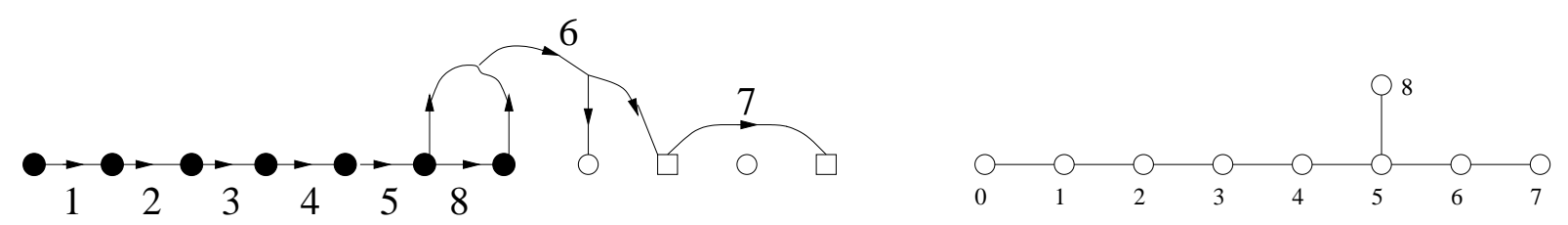

Figure 10: $\widehat{\mathbf{E}}_{8}$ brane configuration and $\widehat{E}_{8}$ Dynkin diagram.

We now consider in detail the special cases:

- $\widehat{\tilde{\mathbf{E}}}_{\mathbf{0}}$ : This configuration consists of three 7-branes $\mathbf{X}_{[\mathbf{2},-\mathbf{1}]} \mathbf{X}_{[-\mathbf{1 , 2}]} \mathbf{X}_{[\mathbf{1}, \mathbf{1}]}(\mathrm{eq}(3.10)$ of [12] with last two branes interchanged). Therefore $K\left(\widehat{\mathbf{E}}_{\mathbf{0}}\right)=T^{9}$ and $\operatorname{Stab}(K)=\{-\mathbb{1}, T\}$. An important property of this configuration is that the asymptotic charge $(p, q)$ of every junction satisfies the condition $p-q \equiv 0(\bmod 3)$. An argument similar to the one used for $\mathbf{D}_{\mathbf{0}}$ proves that if $T^{k} \in \mathcal{D}(w)$ then $k \equiv 0(\bmod 3)$. By explicit computation one can show that $T^{3} \in \mathcal{D}(w):$

$$
\mathbf{X}_{[\mathbf{2},-\mathbf{1}]} \mathbf{X}_{[-\mathbf{1}, \mathbf{2}]} \mathbf{X}_{[\mathbf{1}, \mathbf{1}]} \stackrel{T^{3}}{\longrightarrow} \mathbf{X}_{[-\mathbf{1},-\mathbf{1}]} \mathbf{X}_{[\mathbf{5}, \mathbf{2}]} \mathbf{X}_{[\mathbf{4}, \mathbf{1}]} \stackrel{P_{2}^{-1} P_{1}^{-1}}{\longrightarrow} \mathbf{X}_{[\mathbf{2},-\mathbf{1}]} \mathbf{X}_{[-\mathbf{1}, \mathbf{2}]} \mathbf{X}_{[\mathbf{1}, \mathbf{1}]}
$$

Thus $\mathcal{D}\left(\widehat{\tilde{\mathbf{E}}}_{\mathbf{0}}\right)=\left\{-\mathbb{1}, T^{3}\right\}$ and $\overline{\mathcal{D}}\left(\widehat{\tilde{\mathbf{E}}}_{\mathbf{0}}\right)=\left\{-\mathbb{1}, T^{3} \mid T^{9}=\mathbb{1}\right\}=\mathbb{Z}_{2} \times \mathbb{Z}_{3}$. This configuration supports no algebra. The only junctions having no asymptotic charges (localized) are multiples of the delta junction

$$
\delta=\mathbf{x}_{[2,-1]}+\mathbf{x}_{[-1,2]}-\mathbf{x}_{[1,1]},
$$

which can be represented as a $(-1,0)$ string circling the branes in the counterclockwise direction. The action of the $T^{3}$ duality transformation on a general junction is found as usual using the brane transpositions in (6.30). Denoting the invariant charges on the $\mathbf{X}_{[\mathbf{2},-\mathbf{1}]}, \mathbf{X}_{[-\mathbf{1}, \mathbf{2}]}$, and $\mathbf{X}_{[\mathbf{1 , 1}]}$ branes by $Q_{1}, Q_{2}$ and $Q_{3}$ respectively, we find that

$$
T^{3}:\left(Q_{1}, Q_{2}, Q_{3}\right) \mapsto\left(Q_{2},-Q_{3},-Q_{1}+3\left(Q_{2}+Q_{3}\right)\right) .
$$


In this notation the delta junction is $(1,1,-1)$, and one readily verifies that $T^{3}$ leaves it invariant. This was expected, since $T^{3}$ leaves invariant the $(-1,0)$ string, and brane transpositions cannot affect a looping string.

- $\widehat{\tilde{\mathbf{E}}}_{\mathbf{1}}$ : Here $K\left(\widehat{\mathbf{E}}_{\mathbf{1}}\right)=T^{8}$ and $\operatorname{Stab}(K)=\{-\mathbb{1}, T\}$. Since this brane configuration has an A-brane a simple computation shows that $T \in \mathcal{D}(w)$ :

$$
\mathbf{A X}_{[\mathbf{2},-\mathbf{1}]} \mathbf{X}_{[-\mathbf{1}, \mathbf{2}]} \mathbf{X}_{[\mathbf{1}, \mathbf{1}]} \stackrel{T}{\longrightarrow} \mathbf{A X}_{[\mathbf{1},-\mathbf{1}]} \mathbf{X}_{[\mathbf{1}, \mathbf{2}]} \mathbf{X}_{[\mathbf{2}, \mathbf{1}]} \stackrel{P_{1} P_{2} P_{3} P_{3} P_{2} P_{1}}{\longrightarrow} \mathbf{A X}_{[\mathbf{2},-\mathbf{1}]} \mathbf{X}_{[-\mathbf{1}, \mathbf{2}]} \mathbf{X}_{[\mathbf{1}, \mathbf{1}]}
$$

Thus $\mathcal{D}\left(\widehat{\tilde{\mathbf{E}}}_{\mathbf{1}}\right)=\{-\mathbb{1}, T\}$ and $\overline{\mathcal{D}}\left(\widehat{\tilde{\mathbf{E}}}_{\mathbf{1}}\right)=\left\{-\mathbb{1}, T \mid T^{8}=\mathbb{1}\right\}=\mathbb{Z}_{2} \times \mathbb{Z}_{8}$. A localized junction on this configuration is specified by the number of $\delta$ loops (the junction in (6.31)) and a $u(1)$ charge $\bar{Q}$. We thus write $\mathbf{J}=m \delta+\bar{Q} \overline{\mathbf{J}}[14]$ where

$$
\overline{\mathbf{J}}=3 \mathbf{a}-\mathbf{x}_{[\mathbf{2},-1]}-\mathbf{x}_{[\mathbf{1}, \mathbf{1}]} .
$$

Under the $T$ transformation the junction $\mathbf{J} \equiv(m, Q)$ transforms as

$$
T:(m, \bar{Q}) \mapsto(m+3 \bar{Q}, \bar{Q})
$$

which follows because the $\mathbf{A}$ brane that must be circled around has invariant charge $3 \bar{Q}$.

Since the monodromy of the configuration is $T^{8}$, we see that

$$
T^{8}:(m, \bar{Q}) \mapsto(m+24 \bar{Q}, \bar{Q}),
$$

should be a transformation that can be generated simply by crossing transformations. Such a tranposition (actually its inverse) was discussed in section 3.2. It is not of Weyl type because there are no real root junctions for this brane configuration.

Indeed, the required transposition is the inverse of that discussed in section 3.2 for the case of $\hat{\tilde{\mathbf{E}}}_{1}$. These transpositions are equivalent to first taking the $\mathbf{A}$ brane clockwise around the other three branes three times. This has the effect of changing the labels of the other three branes as if acted by $T^{3}$. Those branes are then restored to their original labels by performing the transpositions indicated in the second step of (6.30). The first step takes a junction $\mathbf{J}=m \delta+\bar{Q} \overline{\mathbf{J}}$ and adds to it $\left(-3 Q_{A}\right)=-9 \bar{Q}$ delta junctions. This step, while changing the labels of the three rightmost branes, it does not change the invariant charges they have; these are, in the notation used for $\widehat{\tilde{\mathbf{E}}}_{\mathbf{0}}, \bar{Q}(-1,0,-1)$ (see (6.34)). Using (6.32) we see that under the restoring transposition: $\bar{Q}(-1,0,-1) \mapsto \bar{Q}(0,1,-2)=\bar{Q}(-1,0,-1)+\bar{Q} \delta$. Therefore, the complete series of transpositions $\tilde{b}$ adds $(-9+1) \bar{Q}=-8 \bar{Q}$ delta junctions:

$$
\tilde{b}:(m, \bar{Q}) \mapsto(m-8 \bar{Q}, \bar{Q})
$$


Comparing with (6.36) we see that indeed $T^{8}$ has the same effect as the transposition $(\tilde{b})^{-3}$. Since a $T$ duality adds $3 \bar{Q}$ delta junctions, and we can add or remove $8 \bar{Q}$ delta junctions by transpositions, $T^{8}$ is the lowest power of $T$ that is equivalent to a transposition.

- $\widehat{\mathbf{E}}_{\mathbf{1}}$ : Here $K\left(\widehat{\mathbf{E}}_{\mathbf{1}}\right)=T^{8}$. A junction of asymptotic charge $(p, q)$ with support on this configuration satisfies the condition $p+q \equiv 0(\bmod 2)$. Thus if $T^{k} \in \mathcal{D}(w)$ then $k \equiv 0(\bmod 2)$. By explicit computation one can show that $T^{2} \in \mathcal{D}(w)$ :

$$
\mathrm{BCBC} \stackrel{T^{2}}{\longrightarrow} \mathbf{X}_{[-\mathbf{1},-\mathbf{1}]} \mathbf{X}_{[\mathbf{3}, \mathbf{1}]} \mathbf{X}_{[-\mathbf{1},-\mathbf{1}]} \mathbf{X}_{\mathbf{3}, \mathbf{1}]} \stackrel{P_{3}^{-1} P_{1}^{-1}}{\longrightarrow} \mathrm{BCBC} .
$$

Thus $\mathcal{D}\left(\widehat{\mathbf{E}}_{\mathbf{1}}\right)=\left\{-\mathbb{1}, T^{2}\right\}$ and $\overline{\mathcal{D}}\left(\widehat{\mathbf{E}}_{\mathbf{1}}\right)=\left\{-\mathbb{1}, T^{2} \mid T^{8}=\mathbb{1}\right\}=\mathbf{Z}_{2} \times \mathbf{Z}_{4}$. The $T^{2}$ transformation acts on the invariant charges in the following way,

$$
T^{2}:\left(Q_{B_{1}}, Q_{C_{1}}, Q_{B_{2}}, Q_{C_{2}}\right) \mapsto\left(Q_{C_{1}},-Q_{B_{1}}+2 Q_{C_{1}}, Q_{C_{2}},-Q_{B_{2}}+2 Q_{C_{2}}\right) .
$$

The invariant charges of the root junctions and the delta junction $\delta$ are 12

$$
\boldsymbol{\alpha}_{\mathbf{0}}=(1,0,-1,0), \boldsymbol{\alpha}_{\mathbf{1}}=(0,1,0,-1), \delta=\boldsymbol{\alpha}_{0}+\boldsymbol{\alpha}_{1}=(1,1,-1,-1) \text {. }
$$

Thus from (6.39) and (6.40) it follows that

$$
T^{2}:\left(\alpha_{0}, \alpha_{1}\right) \rightarrow\left(\alpha_{0}-\delta, \alpha_{1}+\delta\right) .
$$

$\Gamma\left(\widehat{A}_{1}\right)=\mathbf{Z}_{2}$ where the nontrivial element is $\mathcal{O}\left(\alpha_{0}, \alpha_{1}\right)=\left(\alpha_{1}, \alpha_{0}\right)$. One can verify that acting on the simple roots

$$
T^{2}=\mathcal{O} W_{0}
$$

Therefore, $\phi: \overline{\mathcal{D}}\left(\widehat{\mathbf{A}}_{\mathbf{1}}\right) \mapsto \mathbf{Z}_{2} \times \Gamma\left(\widehat{A}_{1}\right)$, is determined by

$$
h\left(T^{2}\right)=\mathcal{O} .
$$

This completes our analysis of the duality groups of affine exceptional brane configurations.
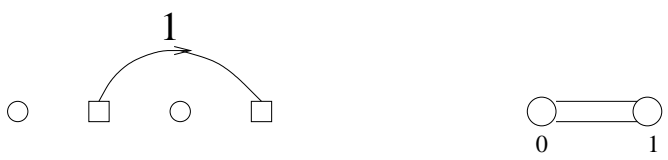

Figure 11: $\widehat{\mathbf{E}}_{\mathbf{1}}$ brane configuration and $\widehat{A}_{1}$ Dynkin diagram. 


\begin{tabular}{|c|l|c|c|c|c|}
\hline$w$ & $K(w)$ & $\mathcal{D}(w)$ & $\overline{\mathcal{D}}(w)$ & $\mathcal{G}$ & $\operatorname{Aut}(Q) / W$ \\
\hline$\widehat{\mathbf{E}}_{\mathbf{0}}$ & $T^{9}$ & $\left\{-\mathbb{1}, T^{3}\right\}$ & $\mathbf{Z}_{2} \times \mathbf{Z}_{3}$ & -- & -- \\
\hline$\widehat{\mathbf{E}}_{\mathbf{1}}$ & $T^{8}$ & $\left\{-\mathbb{1}, T^{2}\right\}$ & $\mathbf{Z}_{2} \times \mathbf{Z}_{4}$ & $\widehat{A}_{1}$ & $\mathbf{Z}_{2} \times \mathbf{Z}_{2}$ \\
\hline$\widehat{\widetilde{\mathbf{E}}}_{\mathbf{1}}$ & $T^{8}$ & $\{-\mathbb{1}, T\}$ & $\mathbf{Z}_{2} \times \mathbf{Z}_{8}$ & $\widehat{u(1)}$ & -- \\
\hline$\widehat{\mathbf{E}}_{\mathbf{2}}$ & $T^{7}$ & $\{-\mathbb{1}, T\}$ & $\mathbf{Z}_{2} \times \mathbf{Z}_{7}$ & $\widehat{A}_{1} \oplus \widehat{u(1)}$ & $\mathbf{Z}_{2} \times \mathbf{Z}_{2}$ \\
\hline$\widehat{\mathbf{E}}_{\mathbf{3}}$ & $T^{6}$ & $\{-\mathbb{1}, T\}$ & $\mathbf{Z}_{2} \times \mathbf{Z}_{6}$ & $\widehat{A}_{2} \oplus \widehat{A}_{1}$ & $\mathbf{Z}_{2} \times\left(\mathcal{D}_{6} \times \mathbf{Z}_{2}\right)$ \\
\hline$\widehat{\mathbf{E}}_{\mathbf{4}}$ & $T^{5}$ & $\{-\mathbb{1}, T\}$ & $\mathbf{Z}_{2} \times \mathbf{Z}_{5}$ & $\widehat{A}_{4}$ & $\mathbf{Z}_{2} \times \mathcal{D}_{10}$ \\
\hline$\widehat{\mathbf{E}}_{\mathbf{5}}$ & $T^{4}$ & $\{-\mathbb{1}, T\}$ & $\mathbf{Z}_{2} \times \mathbf{Z}_{4}$ & $\widehat{D}_{5}$ & $\mathbf{Z}_{2} \times \mathcal{D}_{8}$ \\
\hline$\widehat{\mathbf{E}}_{\mathbf{6}}$ & $T^{3}$ & $\{-\mathbb{1}, T\}$ & $\mathbf{Z}_{2} \times \mathbf{Z}_{3}$ & $\widehat{E}_{6}$ & $\mathbf{Z}_{2} \times \mathcal{D}_{6}$ \\
\hline$\widehat{\mathbf{E}}_{\mathbf{7}}$ & $T^{2}$ & $\{-\mathbb{1}, T\}$ & $\mathbf{Z}_{2} \times \mathbf{Z}_{2}$ & $\widehat{E}_{7}$ & $\mathbf{Z}_{2} \times \mathbf{Z}_{2}$ \\
\hline$\widehat{\mathbf{E}}_{\mathbf{8}}$ & $T$ & $\{-\mathbb{1}, T\}$ & $\mathbf{Z}_{2}$ & $\widehat{E}_{8}$ & $\mathbf{Z}_{2}$ \\
\hline$\widehat{\mathbf{E}}_{\mathbf{9}}$ & $\mathbb{1}$ & $S L(2, \mathbf{Z})$ & $P S L(2, \mathbf{Z})$ & $\widehat{E}_{9}$ & $?$ \\
\hline
\end{tabular}

Table 6: Duality groups and graph automorphisms of $\widehat{\mathbf{E}}_{\mathbf{N}}$ configurations. $\mathcal{D}_{6}=S_{3}$ is the permutation group of three objects and $\mathcal{D}_{2 N}$, the dihedral group, is the group of symmetries of the regular $n$-gon. 


\section{Acknowledgements}

We wish to acknowledge useful conversations with Y. Imamura and V. Kac.

This research was supported in part by the US Department of Energy under contract \#DEFC02-94ER40818.

\section{References}

[1] N. Seiberg and E. Witten, Electric - Magnetic Duality, Monopole Condensation, And Confinement In N=2 Supersymmetric Yang-Mills Theory, Nucl. Phys. B426 (1994) 19, hep-th/9407087;

N. Seiberg and E. Witten, Monopoles, Duality And Chiral Symmetry Breaking In N=2 Supersymmetric QCD, Nucl. Phys. B431 (1994) 484, hep-th/9408099.

[2] A. Sen, F-theory and Orientifolds, Nucl. Phys. B475, 562 (1996), hep-th/9605150.

[3] T. Banks, M.R. Douglas and N. Seiberg, Probing F-theory with branes, Phys. Lett. B387, 278 (1996), hep-th/9605199.

[4] Y. Imamura, E8 Flavour Multiplets, Phys. Rev. D58 (1998) 106005, hep-th/9802189.

[5] O. DeWolfe and B. Zwiebach, String junctions for arbitrary Lie algebra representations Nucl. Phys. B541 (1999) 509-565, hep-th/9804210.

[6] O. DeWolfe, T. Hauer, A. Iqbal and B. Zwiebach, Constraints On The BPS Spectrum Of N=2, D=4 Theories With A-D-E Flavor Symmetry, Nucl. Phys. B534 (1998) 261-274, hep-th/9805220.

[7] A. Mikhailov, N. Nekrasov, S. Sethi, Geometric Realizations of BPS States in N=2 Theories, Nucl. Phys. B531 (1998) 345-362, hep-th/9803142.

[8] O. Bergman, A. Fayyazuddin, String Junctions and BPS States in Seiberg-Witten Theory, Nucl. Phys. B531 (1998) 219-232, hep-th/9802033;

O. Bergman, A. Fayyazuddin, String Junction Transitions in the Moduli Space of $\mathcal{N}=2$ SYM, Nucl. Phys. B535 (1998) 139-151, hep-th/9806011.

[9] M. R. Gaberdiel, T. Hauer, B. Zwiebach, Open string-string junction transitions, Nucl. Phys. B525 (1998) 117, hep-th/9801205.

[10] O. DeWolfe, T. Hauer, A. Iqbal and B. Zwiebach, Uncovering Symmetries on [p.q] 7-branes: Beyond The Kodaira Classification, hep-th/9812028. 
[11] O. DeWolfe, Affine Lie Algebras, String Junctions And 7-Branes, Nucl. Phys. B550 622-637, hep-th/9809026.

[12] O. DeWolfe, T. Hauer, A. Iqbal and B. Zwiebach, Affine and Indefinite Kac-Moody Symmetries on [p,q] 7-branes, hep-th/9812128.

[13] Y. Yamada, and S.-K. Yang Affine 7-brane Backgrounds and Five-Dimensional $E_{N}$ Theories on $S^{1}$, hep-th/9907134.

[14] A. Sen and B. Zwiebach, Stable non-BPS states in F-theory, hep-th/9907164.

[15] T. Hauer, A. Iqbal, Del Pezzo Surfaces and Affine 7-brane Backgrounds, J. High Energy Phys, 01 (2000) 043, hep-th/9910054.

[16] W. Lerche, On the Heterotic/F-Theory Duality in Eight Dimensions, hep-th/9910207.

[17] M. C. Daflon Barrozo, Map of Heterotic and Type IIB Moduli in 8 Dimensions, hepth/9909178.

[18] Y. Imamura, String Junctions and Their Duals in Heterotic String Theory, Prog.Theor.Phys. 101 (1999) 1155-1164, hep-th/9901001.

[19] M. R. Gaberdiel, B. Zwiebach, Exceptional groups from open strings, Nucl. Phys. B518 (1998) 151, hep-th/9709013.

[20] V. Kac, Infinite-dimensional Lie Algebras, Cam. Univ. Press, New York, 1985.

[21] J. E. Humphreys, Introduction to Lie Algebras and Representation Theory, Springer Verlag, New York, 1980. 